\title{
Symplectic tracking and compensation of dynamic field integrals in complex undulator structures
}

\author{
Johannes Bahrdt* and Godehard Wüstefeld \\ Helmholtz-Zentrum Berlin, Albert-Einstein-Straße 15, 12489 Berlin, Germany
}

(Received 31 January 2011; published 6 April 2011)

\begin{abstract}
In first approximation storage ring multipole magnets are described as simple two-dimensional magnet structures and many linear and nonlinear beam optic features of a magnet lattice can already be derived from this model. In contrast, undulators, and in particular variably polarizing devices, employ complicated three-dimensional magnetic fields which may have a severe impact on the electron beam, in particular, in low energy third generation storage rings. A Taylor expanded generating function method is presented to generate a fast, flexible, and symplectic mapping routine for particle tracking in magnetic fields. This method is quite general and is based on the solution of the Hamilton-Jacobi equation. It requires an analytical representation of the fields, which can be differentiated and integrated. For undulators of the APPLE II type, an accurate analytic field model is derived which is suitable for the tracking routine. This field model is fully parametrized representing all operation modes for the production of elliptical or linear polarized light with an arbitrary inclination angle or even arbitrary polarization. Based on this field model, analytic expressions for 2 nd order kicks are derived. They are used to estimate the influence of APPLE II undulators on the electron beam dynamic. Furthermore, an analytic model for the description of shims is given. The shims are needed for field and performance optimization. Passive and active shimming concepts for the compensation of linear and nonlinear effects of variably polarizing undulators are discussed.
\end{abstract}

DOI: $10.1103 /$ PhysRevSTAB.14.040703

PACS numbers: 29.20.db, 07.85.Qe

\section{INTRODUCTION}

Planar undulators for the production of synchrotron radiation have been built for more than 30 years. The vertical focusing is well understood and the linear term can be compensated with an appropriate tuning of storage ring quadrupoles. The linear term of a horizontal defocusing can be compensated with ring quadrupoles as well. Higher order terms can be minimized either by choosing an appropriate undulator pole width or a special transverse pole shape or by implementing additional correction magnets at the undulator ends [1]. A superconducting double helix undulator has been used already in the first FEL experiments at SLAC $[2,3]$. This design provides photons with a fixed helicity. It cannot be operated in a storage ring where a larger horizontal aperture for injection is required. Planar helical or elliptical devices have to be used instead. Over the past 20 years, various permanent magnet based undulator designs for the production of elliptically polarized light have been proposed and built: The Helios design as built at the ESRF [4] employs a functional separation for the field production. Upper and lower magnet girders produce pure horizontal and vertical on-axis fields,

\footnotetext{
*Corresponding author.

Johannes.Bahrdt@Helmholtz-Berlin.de

Published by American Physical Society under the terms of the Creative Commons Attribution 3.0 License. Further distribution of this work must maintain attribution to the author(s) and the published article's title, journal citation, and DOI.
}

respectively. With the implementation of an additional longitudinal movement of one of the girders any state of polarization is realized. Because of the functional separation the independent focusing of upper and lower beam produces an electron beam steering which scales inversely with the square of the electron energy. It can be tolerated at the ESRF $6 \mathrm{GeV}$ machine. The ELETTRA design [5] delivers circularly polarized light at all magnet gaps. However, other states of polarization are not accessible. Usually, elliptically polarizing devices have the drawback of a small transverse good field region for the horizontal field component. At SPRING-8 [6] a magnet design has been optimized for an enlargement of the good field region sacrificing field amplitude.

The highest magnetic field among all existing planar elliptically polarizing undulators is produced by a so-called advanced polarizing photon light emitter (APPLE II) which has been proposed by Sasaki [7]. Today, most of the third generation storage rings rely on the APPLE II design for variably polarizing photon sources. The flexibility of this device provides also other operation modes such as linear polarized light with an inclination with respect to the midplane. Any arbitrary polarization ellipse can be produced if more degrees of freedom are introduced in the mechanic design (see Sec. III A for universal mode). The small transverse good field region of APPLE II undulators has a severe impact on the electron beam dynamics of low electron energy rings which has to be minimized.

Starting from the APPLE II design more sophisticated undulators with even higher fields have been developed for 
the use in fourth generation light sources such as freeelectron lasers (FELs) or energy recovery linacs. These light sources do not require a large horizontal aperture for injection. Hence, more compact designs like the APPLE III [8] or DELTA undulator [9] can be realized. Being single pass machines, the multipole requirements are relaxed as compared to a storage ring.

The operation of undulators in a low energy third generation electron storage ring requires a thorough understanding of the impact of theses devices to the electron beam dynamic. In contrast to conventional accelerator magnets, undulators have a complicated three-dimensional field distribution and the dynamic behavior is dominated by effects introduced by the oscillating electron trajectory caused by the periodic undulator fields. These effects are usually called dynamic kicks or 2nd order kicks because they scale inversely with the square of the particle energy. They cannot be derived from straight line field integrals and, hence, they cannot directly be measured by moving wires, pulsed wires, rotating coils, search coils, or Hall probes. In contrast, the so-called vibrating wire method is capable to measure the field integrals along a wiggling trajectory [10]. The wire is powered with a DC current and the Lorentz-force deforms the wire in a periodic manner mimicking the electron trajectory. On the other hand, the dynamic field integrals can be evaluated with an accuracy of a few percent from the measured undulator field.

The electron beam dynamic is studied in tracking simulations where electron trajectories through the accelerator magnets are evaluated for thousands of subsequent turns. Tracking codes use one of two approaches or a combination of both to construct the magnetic field: (1) The fields are described explicitly in terms of measured or evaluated field maps. (2) Analytic models are used for the description of the fields. The pure numeric approach (1) determines the electron trajectory from a symplectic twofold integration of the magnetic fields. This approach is adequate for devices with only a few poles such as wavelength shifters but it is less efficient for undulators with dozens of periods because several integration steps are needed for each undulator period. The analytic approach (2) offers the chance to perform an analytic integration. The accuracy is determined by the quality of the model (expansion order and integration step size). For a comparable accuracy, the speed can be significantly faster as compared to the numeric algorithm because the step size can be as large as many undulator periods. This allows for a detailed scanning of a large parameter space with a reasonable effort.

In Sec. II we review an analytic, symplectic and fast tracking algorithm as developed at BESSY. The fast tracking algorithm is based on analytic field descriptions. The analytic field model includes a full parametrization with respect to various operation parameters of the insertion devices (ID). Thus, tracking in a wide parameter space is decoupled from field simulations. This is in contrast to methods where the insertion device is represented by a kick map [11]. These maps have to be evaluated for each operation mode which can be time consuming in particular for complicated magnet structures such as helical or elliptical undulators.

In Sec. III we present analytic expressions of the fields of a variably polarizing undulator of the APPLE II type. Though we will concentrate on this device type, the method can easily be adapted to other magnet designs. The analytic field model is used in the tracking algorithm of Sec. II.

Based on the analytic field description of Sec. III we derive an analytic model of 2nd order kicks in APPLE II type undulators in Sec. IV. It will be discussed that, principally, 2nd order kicks cannot be compensated with usual shims. However, their influence on beam dynamics can significantly be reduced.

In low energy rings such as BESSY II the dynamic field integrals are shimmed to minimize the impact of the undulators in terms of lifetime and injection efficiency. In the beam decaying mode every several hours the undulator gaps are opened for injection. A long lifetime keeps the number of injections low. In the top-up mode electrons are injected every few minutes with undulator gaps closed. A high injection efficiency is crucial in this mode. For realistic tracking studies the shims have to be implemented into the model. In Sec. V an analytical parametrization of passive and active shims is given which is used in the tracking scheme of Sec. II. The shimming techniques used at BESSY II are described.

\section{SYMPLECTIC PARTICLE TRACKING BASED ON GENERATING FUNCTIONS}

In this section, a Taylor expanded generating function (GF) is presented, to generate a symplectic mapping routine for particle tracking in magnetic fields. It is an iterative solution of the Hamilton-Jacobi equation. This method is quite general, extremely fast, and very flexible. It requires an analytical representation of the magnetic vector potential, which can be differentiated and integrated. This analytic vector potential can, for example, be derived from a Fourier series expansion of the magnetic fields (see Sec. III). The method shown here is a series expansion of the GF with respect to the particle momenta. For tracking the 2nd order is applied. It can be extended to higher orders. The tracking routine is given in an explicit form, transforming the initial canonical variables to the final variables. This method is very well suited for fields, described by "Halbach"-type field harmonics (see Sec. III), commonly applied to wigglers and undulators.

The method of numerical and analytical based approximated GF is applied at BESSY II for particle tracking passing insertion devices. This method goes back to [12], where a numerical based GF was applied to simulate full turn, transverse beam dynamics in fixed field alternating gradient (FFAG) accelerators. Here we are specifically 
interested in an analytical GF over a limited section of the ring, such as wigglers or undulators. Applications of this method are published in several conference proceedings. In $[13,14]$ the GF for a planar undulator was presented. In [15] a general method for a GF of magnetic fields was derived, and in [16] the Hamilton-Jacobi method was applied to derive an iterative solution for the GF. Helical undulators are discussed in $[17,18]$, with special emphasis on entrance and exit poles [19]. For a numerical fit of a Taylor expanded GF with respect to transverse position and momentum variables, see [20]. This section is intended to summarize the method of constructing the GF for particle tracking from analytical descriptions of the magnetic fields. A type of GF is chosen, commonly called $F_{3}$, which results in an explicit, approximated transformation, of very good accuracy.

Publications of other authors on GF for particle tracking are not discussed here. However, there are at least two early papers which should be mentioned, which present applications of the GF. In one paper a method of symplectic integration is derived [21], in the other one a GF expansion in action and angle variables is applied for studying full turn maps of a storage ring [22]. For simulations of undulator and wiggler effects also a kick map is often applied, as developed, for example, by [11].

\section{A. A Taylor expanded generating function}

We describe the particle motion in a Cartesian coordinate system with $x, y$, and $z$ as the horizontal, vertical, and longitudinal direction, respectively. The Hamiltonian, a function of the local particle variables of a particle in a magnetic field, is given by [23]:

$$
\tilde{H}=\sqrt{(\vec{p}-e \vec{A})^{2} c^{2}+m_{0}^{2} c^{4}},
$$

where $\vec{p}=\left(\tilde{p}_{x}, \tilde{p}_{y}, \tilde{p}_{z}\right)$ is the canonical particle momentum and $\vec{A}=\left(\tilde{A}_{x}, \tilde{A}_{y}, \tilde{A}_{z}\right)$ the vector potential of the magnetic field. A new Hamiltonian, which is the negative of the longitudinal momentum [23], can be defined in a form, which is more convenient for particle tracking. It describes the transverse canonical particle variables $\left(x, p_{x}, y, p_{y}\right)$ in phase space with the longitudinal position $z$ as independent variable. This Hamiltonian is derived by solving the Hamiltonian of Eq. (1) for $-p_{z}$ and a Taylor series expansion of the square root with respect to $p_{x}, p_{y}$ and $A_{x}, A_{y}$ up to second order [23],

$$
H=-p_{z}=-1+\left(p_{x}-A_{x}\right)^{2} / 2+\left(p_{y}-A_{y}\right)^{2} / 2-A_{z},
$$

where we normalized the momenta by the total particle momentum $p_{0}=\sqrt{\tilde{H}^{2} / c^{2}-m_{0}^{2} c^{2}}, \quad p_{x}=\tilde{p}_{x} / p_{0}, \quad p_{y}=$ $\tilde{p}_{y} / p_{0}, p_{z}=\tilde{p}_{z} / p_{0}$ and the vector potential by the magnetic rigidity $B \rho, A_{x}=\tilde{A}_{x} / B \rho, A_{y}=\tilde{A}_{y} / B \rho, A_{z}=$ $\tilde{A}_{z} / B \rho$. These normalized expressions are in general small quantities and can be used as expansion variables. The deviation of the particle from the nominal momentum is taken into account by different $B \rho$ and $p_{0}$ values.

Between the canonical momenta $\left(p_{x}, p_{y}, p_{z}\right)$ and the slope of the trajectory with respect to the beam reference axis $\theta_{x}=d x / d z=x^{\prime}$ and $\theta_{y}=d y / d z=y^{\prime}$, the following relation holds:

$$
\begin{aligned}
& p_{x}=\theta_{x} / \sqrt{1+\theta_{x}^{2}+\theta_{y}^{2}}+A_{x}, \quad \text { and } \\
& p_{y}=\theta_{y} / \sqrt{1+\theta_{x}^{2}+\theta_{y}^{2}}+A_{y},
\end{aligned}
$$

and from the canonical momenta the kinematic momenta $\left(p_{x}^{(k)}, p_{y}^{(k)}, p_{z}^{(k)}\right)$ are calculated (normalized by $\left.p_{0}\right)$,

$$
p_{x}^{(k)}=p_{x}-A_{x}, \quad p_{y}^{(k)}=p_{y}-A_{y},
$$

yielding the slopes as

$$
\begin{aligned}
& \theta_{x}=\frac{p_{x}^{(k)}}{p_{z}^{(k)}}=p_{x}^{(k)} / \sqrt{1-p_{x}^{(k) 2}-p_{y}^{(k) 2}} \text { and } \\
& \theta_{y}=\frac{p_{y}^{(k)}}{p_{z}^{(k)}}=p_{y}^{(k)} / \sqrt{1-p_{x}^{(k) 2}-p_{y}^{(k) 2}}
\end{aligned}
$$

For small momenta we approximate $\theta_{x}=p_{x}^{(k)}$ and $\theta_{y}=$ $p_{y}^{(k)}$. The expansion of the Hamiltonian of Eq. (1) into the form of Eq. (2) can be done with respect to five dimensionless variables, $\left(p_{x}, p_{y}, A_{x}, A_{y}, A_{z}\right)$. We can summarize the expansion by $\left(A_{x}, A_{y}, A_{z}\right)$ by the following trick. We introduce a new variable $x_{3}$, attached as a factor to $A_{x}, A_{y}$, and $A_{z}$, which counts the order of these vector potential terms. For the linear terms we have $A_{x} \rightarrow A_{x} x_{3}$, $A_{y} \rightarrow A_{y} x_{3}, A_{z} \rightarrow A_{z} x_{3}$, for the second order terms, for example, $A_{x}^{2} \rightarrow A_{x}^{2} x_{3}^{2}, A_{x} A_{y} \rightarrow A_{x} A_{y} x_{3}^{2}$, and so on. We will use $x_{3}$ like an expansion variable, and in the final result replace it by $x_{3}=1$. The components of the vector potential become part of the coefficient terms. Then, the Hamiltonian of Eq. (2) can be derived from Eq. (1) by expansion with respect to three variables, $\left(p_{x}, p_{y}, x_{3}\right)$. From the Hamiltonian Eq. (2) the differential increment of the variables is given by

$$
\begin{aligned}
x^{\prime} & =\partial H / \partial p_{x} & y^{\prime} & =\partial H / \partial p_{y} \\
p_{x}{ }^{\prime} & =-\partial H / \partial x & p_{y}{ }^{\prime} & =-\partial H / \partial y,
\end{aligned}
$$

where the prime indicates the change of the variable with respect to the longitudinal $z$ position, e.g. $x^{\prime}=d x / d z$. A given set of initial variables can be uniquely transferred along a specified longitudinal distance by integration of Eq. (6) to a set of final variables, by applying Hamiltonian mechanics,

$$
\left(x, p_{x}, y, p_{y}\right) \rightarrow\left(x_{f}, p_{x f}, y_{f}, p_{y f}\right) .
$$

This is a commonly applied variable transformation. Any other appropriate subset of variables can be chosen, such as initial position and final momenta, 


$$
\left(x, p_{x f}, y, p_{y f}\right) \rightarrow\left(x_{f}, p_{x}, y_{f}, p_{y}\right),
$$

and completed (in a more complicated way) to the full set by Hamiltonian mechanics. This last variable set is of interest for the present discussion.

Numerical integration methods are commonly applied to calculate the particle trajectories inside magnetic fields. To perform the calculation, the differential steps of Eq. (6) are approximated by various methods as difference steps, e.g. $x^{\prime}=d x / d z \rightarrow \Delta x / \Delta z$, for numerical orbit integration. Because Eq. (6) shows differential expressions, these difference approximations could lead to nonphysical behavior and require correction methods [21]. But in any case, a small step length $\Delta z$ is required. Instead of using the transformation of Eq. (6), one might perform first the integration of $H$ with respect to $z$ and then directly derive the new particle variables. This method is developed by means of generating functions (GFs). In general, the GF is not known, different to the Hamiltonian, beside special cases. Here, we present a general, but iterative method to construct the GF. This method requires that the fields can be well described by mathematical functions, which can be integrated and differentiated. The advantage of using the GF is that it leads to a symplectic transformation for arbitrary step lengths. However, in the presented method the step length is still involved, it limits the accuracy of the applied Taylor approximation, but the steps could be chosen much longer than by the integration method. The GF describes phase space variables of two phase spaces which are connected by a canonical transformation. One of the special features of the GF is that it mixes variables from the initial phase space with variables from final phase space. It transforms, for example, the initial position and the final momenta to the initial momenta and final positions, independent on the chosen step length.

To derive the GF we start with the Hamilton-Jacobi equation (HJE) [24]. This equation states that the partial derivative of the GF, $F$, with respect to the longitudinal position yields the Hamiltonian

$$
\partial F / \partial z=-H .
$$

This equation demonstrates that the GF is closely linked to the Hamiltonian. There are several types of GF which could be chosen. Here we take the GF of type $F=F_{3}$, which is a function of the initial position and final momenta, $F_{3}=F_{3}\left(x, p_{x f}, y, p_{y f}\right)$. The reason for this choice will become clear later on. As a property of the GF the following particle variables can be derived from it:

$$
\begin{array}{ll}
x_{f}=-\partial F_{3} / \partial p_{x f} & y_{f}=-\partial F_{3} / \partial p_{y f} \\
p_{x}=-\partial F_{3} / \partial x & p_{y}=-\partial F_{3} / \partial y .
\end{array}
$$

This is the corresponding form of Eq. (6), but now the variables are derived directly from $F_{3}$. Only, if the GF is dependent on the correct set of variables, it satisfies these relations. It is the partial derivative of $F_{3}$ with respect to $z$ which is required for the HJE, not the total one, see Eq. (9). This implies that the reverse operation, to construct the GF from the Hamiltonian, would be the integration along $z$ at fixed transverse positions, formally written as

$$
\begin{aligned}
F_{3} & =-\int H d z \\
& =-\int\left[1-\left(p_{x}-A_{x}\right)^{2} / 2+\left(p_{y}-A_{y}\right)^{2} / 2-A_{z}\right] d z+\tilde{F},
\end{aligned}
$$

where $\tilde{F}=\tilde{F}\left(x, p_{x f}, y, p_{y f}\right)$ is a $z$ independent integration constant, which has to be defined. All integrations are performed over the considered step length range.

If the Hamiltonian is derived from the GF as the HJE (9) shows, it would be also a function of the local position and the final momenta, $H=H\left(x, p_{x f}, y, p_{y f}\right)$. This Hamiltonian, expressed by these variables, is not known. Normally, the Hamiltonian is given as a function of the local variables, see Eq. (2), where the momenta and the vector potential are depending on the local position variables. We can construct the Hamiltonian with the required variables by applying the properties of $F_{3}: p_{x}=$ $-\partial F_{3} / \partial x=-F_{3 x}, p_{y}=-\partial F_{3} / \partial y=-F_{3 y}$ which gives

$$
\begin{aligned}
H\left(x, p_{x}, y, p_{y}\right) & \rightarrow H\left(x, p_{x}=-F_{3 x}, y, p_{y}=-F_{3 y}\right) \\
& =H\left(x, p_{x f}, y, p_{y f}\right) .
\end{aligned}
$$

This yields the GF and the Hamiltonian in the same, correct variables set,

$$
H\left(x, p_{x f}, y, p_{y f}\right)+\partial F_{3}\left(x, p_{x f}, y, p_{y f}\right) / \partial z=0 .
$$

If the momenta in the HJE are replaced by $F_{3 x}$ and $F_{3 y}$, we achieve a first order differential equation for $F_{3}$, by applying the Hamiltonian of Eq. (2), now including the order counting variable $x_{3}$,

$$
-1+\left(F_{3 x}+A_{x} x_{3}\right)^{2} / 2+\left(F_{3 y}+A_{y} x_{3}\right)^{2} / 2-A_{z} x_{3}+F_{3 z}=0,
$$

with $\partial F_{3} / \partial z=F_{3 z}$. This differential equation depends on the minimal set of coordinate variables; they are independent on each other. To solve this equation, we choose as an ansatz a Taylor series expansion of the GF of the following type:

$$
F_{3}=\sum_{i j k} f_{i j k} p_{x}^{i} p_{y f}^{j} x_{3}^{k} .
$$

The $f_{i j k}$ coefficients are functions of the local position variables $x, y, z$, the expansion order is given by $i+j+k$. A partial derivative of $f_{i j k}$ with respect to the position variables will be indicated by an additional variable index. An algebraic routine [25] which solves an equation as in Eq. (14) for arbitrary vector potentials $\left(A_{x}, A_{y}, A_{z}\right)$ by iteration is given in [16] for the similar equation based 
on $F_{2}$. For the present choice of $F_{3}$ the algebraic routine is given in Appendix A.

\section{B. Transformations in drift sections}

The first terms of Eq. (15) are defined by the drift transformation. In this case there are no vector fields, there are only $f_{i j k}$ terms involved with $k=0$, and the Hamiltonian of Eq. (2) becomes

$$
H=-1+p_{x}^{2} / 2+p_{y}^{2} / 2 .
$$

From $\partial H / \partial x=-p_{x}{ }^{\prime}=0$ (and similar for $p_{y}{ }^{\prime}$ ) follows that the momentum is constant, and we can replace $p_{x}=p_{x f}$ and $p_{y}=p_{y f}$ to express the GF of Eq. (11) in the correct variables and directly integrate over the step length $z_{f}$ :

$$
\begin{aligned}
F_{3} & =-\int\left(-1+p_{x f}^{2} / 2+p_{y f}^{2} / 2\right) d z+\tilde{F} \\
& =z_{f}-z_{f}\left(p_{x f}^{2}+p_{y f}^{2}\right) / 2-\left(x p_{x f}+y p_{y f}\right),
\end{aligned}
$$

where we added the integration constant $\tilde{F}=-\left(x p_{x f}+\right.$ $\left.y p_{y f}\right)$. This integration constant is $z$ independent and introduces the position variables $(x, y)$ at the starting point. From this solution a first set of expansion coefficients is derived:

$$
\begin{array}{lll}
f_{000}=z_{f} & f_{100}=-x & f_{010}=-y \\
f_{200}=-z_{f} / 2 & f_{110}=0 & f_{020}=-z_{f} / 2 .
\end{array}
$$

From the solution for the drift the conjugated variables are derived,

$$
\begin{aligned}
& x_{f}=-\partial F_{3} / \partial p_{x f}=x+p_{x f} z_{f} \\
& p_{x}=-\partial F_{3} / \partial x=p_{x f} \\
& y_{f}=-\partial F_{3} / \partial p_{y f}=y+p_{y f} z_{f} \\
& p_{y}=-\partial F_{3} / \partial y=p_{y f},
\end{aligned}
$$

which can be arranged to an explicit mapping routine. These expansion coefficients are independent on the magnetic field, they stay unchanged if field dependent terms are included.

\section{Transformations in magnetic fields}

Further $f_{i j k}$ terms of the GF are derived by inserting the expanded expression of the GF of Eq. (15) into Eq. (14) and comparing coefficients of equal order in $p_{x f}, p_{y f}$, and $x_{3}$. Up to second order there will be four additional terms, $f_{001}, f_{002}, f_{011}$, and $f_{101}$, describing the interaction with the magnetic field. The GF is then

$$
\begin{aligned}
F_{3}= & z_{f}-\left(p_{x f} x+p_{y f} y\right)-\left(p_{x f}^{2}+p_{y f}^{2}\right) z_{f} / 2+f_{101} p_{x f} x_{3} \\
& +f_{011} p_{y f} x_{3}+f_{002} x_{3}^{2}+f_{001} x_{3}
\end{aligned}
$$

and the partial derivatives become

$$
\begin{aligned}
F_{3 z}= & 1-\left(p_{x f}^{2}+p_{y f}^{2}\right) / 2+f_{101 z} p_{x f} x_{3}+f_{011 z} p_{y f} x_{3} \\
& +f_{002 z} x_{3}^{2}+f_{001 z} x_{3} \\
F_{3 x}= & -p_{x f}+f_{101 x} p_{x f} x_{3}+f_{011 x} p_{y f} x_{3}+f_{002 x} x_{3}^{2} \\
& +f_{001 x} x_{3} \\
F_{3 y}= & -p_{y f}+f_{101 y} p_{x f} x_{3}+f_{011 y} p_{y f} x_{3}+f_{002 y} x_{3}^{2} \\
& +f_{001 y} x_{3} .
\end{aligned}
$$

This is inserted into Eq. (14) of the HJE and, if only up to 2nd order terms are included, the expanded form of the HJE becomes

$$
\begin{aligned}
- & 1+\left(-p_{x f}+f_{001 x} x_{3}+A_{x} x_{3}\right)^{2} / 2 \\
& +\left(-p_{y f}+f_{001 y} x_{3}+A_{y} x_{3}\right)^{2} / 2-A_{z} x_{3} \\
& +1-\left(p_{x f}^{2}+p_{y f}^{2}\right) / 2+f_{101 z} p_{x f} x_{3}+f_{011 z} p_{y f} x_{3} \\
& +f_{002 z} x_{3}^{2}+f_{001 z} x_{3}=0 .
\end{aligned}
$$

By comparing terms of equal order we get

$$
\begin{aligned}
& f_{001 z}=A_{z} \\
& f_{011 z}=f_{001 y}+A_{y} \\
& f_{101 z}=f_{001 x}+A_{x} \\
& f_{002 z}=-\left(f_{001 x}+A_{x}\right)^{2} / 2-\left(f_{001 y}+A_{y}\right)^{2} / 2 .
\end{aligned}
$$

By integration over the $z$ position of the specified step length follows

$$
\begin{aligned}
f_{001}= & \int A_{z} d z \quad f_{101}=\int\left(A_{x}+\int\left(\partial A_{z} / \partial x\right) d z^{\prime}\right) d z \\
f_{011}= & \int\left(A_{y}+\int\left(\partial A_{z} / \partial y\right) d z^{\prime}\right) d z \\
f_{002}= & -(1 / 2) \int\left[\left(A_{x}+\int\left(\partial A_{z} / \partial x\right) d z^{\prime}\right)^{2}\right. \\
& \left.+\left(A_{y}+\int\left(\partial A_{z} / \partial y\right) d z^{\prime}\right)^{2}\right] d z
\end{aligned}
$$

This yields the GF $F_{3}$ up to second order. If required, the numerical convergence can be improved, if the following third order terms are included:

$$
\begin{aligned}
f_{003}= & \frac{1}{2} \int\left[\frac{\partial}{\partial y_{i}}\left(\int A_{y}^{2} d z\right)\right] A_{y} d z+\frac{1}{2} \int\left[\frac{\partial}{\partial x_{i}}\left(\int A_{y}^{2} d z\right)\right] A_{x} d z \\
& +\frac{1}{2} \int\left[\frac{\partial}{\partial y_{i}}\left(\int A_{x}^{2} d z\right)\right] A_{y} d z \\
& +\frac{1}{2} \int\left[\frac{\partial}{\partial x_{i}}\left(\int A_{x}^{2} d z\right)\right] A_{x} d z
\end{aligned}
$$

The $f_{003}$ term is here expressed with the special choice $A_{z}=0$, to simplify the result. The full term can be generated by applying the routine of Appendix A. From this GF $F_{3}$ the variable transformation is derived as shown in Eq. (10) without $x_{3}$ terms: 


$$
\begin{aligned}
& x_{f}=x+p_{x f} z-f_{101} \\
& p_{x}=p_{x f}-f_{101 x} p_{x f}-f_{011 x} p_{y f}-f_{002 x}-f_{001 x} \\
& y_{f}=y+p_{y f} z-f_{011} \\
& p_{y}=p_{y f}-f_{101 y} p_{x f}-f_{011 y} p_{y f}-f_{002 y}-f_{001 y} .
\end{aligned}
$$

The equation system is only of linear character in the momenta, but nonlinear in the position variables. This can be solved for the final variables. Applying the abbreviation $p_{n}=\left(1-f_{011 y}\right)\left(1-f_{101 x}\right)-f_{011 x} f_{101 y}$ for the denominator, we achieve the following explicit mapping routine:

$$
\begin{aligned}
p_{x f}= & {\left[\left(1-f_{011 y}\right)\left(p_{x}+f_{002 x}+f_{001 x}\right)\right.} \\
& \left.+f_{011 x}\left(p_{y}+f_{002 y}+f_{001 y}\right)\right] / p_{n} \\
x_{f}= & x-f_{101}+p_{x f} z_{f} \\
p_{y f}= & {\left[\left(1-f_{101 x}\right)\left(p_{y}+f_{002 y}+f_{001 y}\right)\right.} \\
& \left.+f_{101 y}\left(p_{x}+f_{002 x}+f_{001 x}\right)\right] / p_{n} \\
y_{f}= & y-f_{011}+p_{y f} z_{f} .
\end{aligned}
$$

All the $f_{i j k}$ terms and their derivatives are only dependent on the initial position coordinates of the particle. They can be easily calculated by numerical methods also in the case of complex field descriptions. This was the reason to choose the GF of type $F_{3}$. A higher order expansion only in the $x_{3}$ variable will increase the accuracy of the approximation and still could be solved in a similar way. A higher order expansion in the momenta makes the solution of the equation system more complicated, but still could be solved by a Newton Raphson method. A general method to generate higher order terms is given in Appendix A.

The vector potential is a key function for the calculation of the GF. More common is the scalar potential function of the magnetic field. A relationship between both types of potentials is given here. There is some freedom in defining the vector potential; we choose a form with the gauge $A_{z}=0$; this simplifies the formulas without limiting the validity. The scalar potential is related to the vector potential as

$$
\begin{aligned}
A_{x} & =-\int(\partial V / \partial y) d z+C_{1} \\
A_{y} & =\int(\partial V / \partial x) d z+C_{2} \\
A_{z} & =0 .
\end{aligned}
$$

In this case the integration constants $C_{1}=C_{1}(x, y), C_{2}=$ $C_{2}(x, y)$ need to be specified. They have to be defined in such a way that the magnetic field components $\vec{B}$ are correctly derived from the vector potential, by $\vec{B}=$ $\vec{\nabla} \times \vec{A}$, and agree with the fields derived from the scalar potential $\vec{B}=-\vec{\nabla} V$. This integration is similar but different to the first field integrals $I_{x}=\int_{z_{1}}^{z_{2}}(\partial V / \partial x) d z$ or
$I_{y}=\int_{z_{1}}^{z_{2}}(\partial V / \partial y) d z$. In the case of the field integrals, the integration limits are explicitly defined and there are no integration constants.

As a summary, the achieved mapping routines are approximated, but still physically correct within the Hamiltonian mechanics, i.e., the approximated expansion of the GF is still symplectic. A further advantage of the GF method is that a longer step length compared to integration methods can be applied, which reduces the numerical calculation time. This method is especially useful for particle tracking in wiggler and undulators, where an averaging over fast oscillating fields is achieved. A numerical integration along the particle orbit would require short steps to resolve the field oscillations, whereas the discussed GF averages analytically over the oscillations. The required vector potential terms can be calculated from Fourier harmonics of the magnetic field (see Appendix D for an APPLE II undulator). Typically about 30 harmonics yield a good approximation. One set of harmonics is sufficient to simulate all operation modes of an APPLE II undulator, producing arbitrary states of polarization. The scaling parameter $B \rho$ takes different particle momenta into account.

\section{Applications}

\section{The undulator}

To demonstrate the method we calculate the GF for a planar undulator. For simplicity the undulator is described by a single harmonic Halbach expansion of the scalar field potential with a field strength parameter $B_{0}$,

$$
V=-\left(B_{0} / k_{y}\right) \cos \left(k_{x} x\right) \sinh \left(k_{y} y\right) \cos (k z+\varphi) .
$$

This yields the vector potential

$$
\begin{aligned}
& A_{x}=\left(B_{0} / k_{z}\right) \cos \left(k_{x} x\right) \cosh \left(k_{y} y\right) \sin (k z+\varphi) \\
& A_{y}=\left[\left(B_{0} k_{x}\right) /\left(k_{y} k_{z}\right)\right] \sin \left(k_{x} x\right) \sinh \left(k_{y} y\right) \sin (k z+\varphi) \\
& A_{z}=0 .
\end{aligned}
$$

From the scalar and the vector potential the correct longitudinal $B$ field is derived, if the relation $k_{x}^{2}+k=k_{y}^{2}$ holds. By applying our formulas [Eq. (24)], we get for the field dependent $f_{i j k}$ terms integrated over $N$ multiples of a full period $\lambda_{z}, z_{f}=N \lambda_{z}$,

$$
\begin{aligned}
f_{101}= & 0 \\
f_{011}= & 0 \\
f_{002}= & -z_{f} B_{0}^{2}\left[k_{y}^{2} \cos ^{2}\left(k_{x} x\right) \cosh ^{2}\left(k_{y} y\right)\right. \\
& \left.+k_{x}^{2} \sin ^{2}\left(k_{x} x\right) \sinh ^{2}\left(k_{y} y\right)\right] /\left(2 k_{y} k\right)^{2} .
\end{aligned}
$$

This result is used to derive the transformation of the particle coordinates applying Eq. (27), 


$$
\begin{aligned}
p_{x f}= & p_{x}+f_{002 x} \\
x_{f}= & x+p_{x f} z_{f} \\
p_{y f}= & p_{y}+f_{002 y} \\
y_{f}= & y+p_{y f} z_{f} \\
f_{002 x}= & z_{f} B_{0}^{2} 2 k_{x} \sin \left(k_{x} x\right) \cos \left(k_{x} x\right)\left[k_{y}^{2} \cosh ^{2}\left(k_{y} y\right)\right. \\
& \left.-k_{x}^{2} \sinh ^{2}\left(k_{y} y\right)\right] /\left(2 k_{y} k\right)^{2} \\
f_{002 y}= & -z_{f} B_{0}^{2} 2 k_{y} \cosh \left(k_{y} y\right) \sinh \left(k_{y} y\right)\left[k_{y}^{2} \cos ^{2}\left(k_{x} x\right)\right. \\
& \left.+k_{x}^{2} \sin ^{2}\left(k_{x} x\right)\right] /\left(2 k_{y} k\right)^{2} .
\end{aligned}
$$

The achieved GF is independent on the phase shift term $\varphi$; this dependency did vanish by the integration procedure. This phase shift becomes involved again, if one changes from the canonical momenta $p_{x}, p_{y}$ to $x^{\prime}, y^{\prime}$ where the vector potentials are required, which are still dependent on $\varphi$. The field integrals $\int_{0}^{N \lambda_{z}} B_{y} d z$ and $\int_{0}^{N \lambda_{z}} \int_{0}^{z^{\prime}} B_{y} d z d z^{\prime}$ and similar for $B_{x}$ vanish only, if $\varphi=0$. In this case, the beam distortion by the undulator is minimal. The resulting transformation depends on the number of periods $N$ per step length. For $N=1$ the convergence in most cases is very good. More complicated fields, composed by superposition of higher field harmonics as discussed in Sec. III, can be easily performed by numerical methods. A step length of half, one or more integer periods results in simplified expressions.

In the case of a helical undulator the handling of the phase shift term $\varphi$ is more complicated. A careful matching by end poles is required [19]. Outside of the undulator the reference beam orbit is a straight line with $x=y=0$. Inside of the helical undulator the reference beam orbit is a helical orbit. The transition between these two sections of the reference orbits is performed by the end poles. The end poles are dependent on the tuning of the helical device. A possible approach for the end poles is the following. The undulator consists of the sequence of equal poles of amplitude factors +1 and -1 . There are two poles per period. The $B$ fields oscillate inside the undulator periodically with equal field shape of period length $\lambda_{0}$. The end pole entrance into the undulator starts with the same pole shape, but the first pole has an amplitude strength of $+1 / 4$, the second one a strength of $-3 / 4$. The first pole of the periodic section has a strength of +1 . The exit is done the opposite way around. These end poles can be applied for the helical undulators for any tuning of the phase shifts yielding vanishing field integrals, as required.

\section{Transverse dynamic kicks}

In a second example the transverse momentum kicks of a particle passing through the magnetic field of the undulator are calculated. The vector potentials of the undulator fields performed sinlike oscillations with vanishing average value over multiples of a period. With the choice of $A_{z}=0$ the remaining field dependent terms of $F_{3}$ simplify to

$$
\begin{gathered}
f_{101}=\int_{0}^{z_{f}} A_{x} d z=0 \quad f_{011}=\int_{0}^{z_{f}} A_{y} d z=0, \\
f_{002}=-\int_{0}^{z_{f}}\left(A_{x}^{2}+A_{y}^{2}\right) d z / 2 .
\end{gathered}
$$

Applying the 2nd order expansion, only the $f_{002}$ term stays different from zero. The change of the particle momenta $\Delta p_{x}, \Delta p_{y}$, for an on-axis particle $(x=y=0)$ is then given as

$$
\begin{aligned}
& \Delta p_{x}=p_{x f}-p_{x}=F_{3 x}+p_{x f}=f_{002 x} \\
& \Delta p_{y}=p_{y f}-p_{y}=F_{3 y}+p_{y f}=f_{002 y} .
\end{aligned}
$$

For further discussion of undulator fields we assume a periodically vector potential similar as given in the example of the planar undulator,

$$
A_{x}=a_{x} \sin (k z+\varphi) \quad A_{y}=a_{y} \sin (k z+\varphi) \quad A_{z}=0,
$$

where the transverse potential dependencies are included in the factors $a_{x}(x, y), a_{y}(x, y)$ and an arbitrary phase shift $\varphi$. The magnetic fields $B_{x}$ and $B_{y}$ are then derived as

$$
\begin{aligned}
& B_{x} / B \rho=-\partial A_{y} / \partial z=-a_{y} k \cos (k z+\varphi) \\
& B_{y} / B \rho=\partial A_{x} / \partial z=a_{x} k \cos (k z+\varphi) .
\end{aligned}
$$

The coefficients $a_{x}(x, y) k$ and $a_{y}(x, y) k$ can be considered as the peak value of the magnetic fields $B_{x, 0}(x, y) / B \rho$ and $B_{y, 0}(x, y) / B \rho$ along the undulator period. The integral along the $z$ direction of period length $z_{f}$ is then easily performed,

$$
f_{002}=-\frac{1}{2} \frac{z_{f}}{2}\left(a_{x}^{2}+a_{y}^{2}\right)=-\frac{z_{f}}{(2 k B \rho)^{2}}\left(B_{x, 0}^{2}+B_{y, 0}^{2}\right) .
$$

The transverse canonical momentum kicks of the particle are derived from the transverse derivatives taken at the peak fields,

$$
\begin{aligned}
\Delta p_{x} & =f_{002 x}=-\frac{1}{2} \frac{z_{f}}{2} \partial\left(a_{x}^{2}+a_{y}^{2}\right) / \partial x \\
& =-\frac{z_{f}}{(2 k B \rho)^{2}}\left(2 B_{x, 0} \partial B_{x, 0} / \partial x+2 B_{y, 0} \partial B_{y, 0} / \partial x\right) \\
\Delta p_{y} & =f_{002 y}=-\frac{1}{2} \frac{z_{f}}{2} \partial\left(a_{x}^{2}+a_{y}^{2}\right) / \partial y \\
& =-\frac{z_{f}}{(2 k B \rho)^{2}}\left(2 B_{x, 0} \partial B_{x, 0} / \partial y+2 B_{y, 0} \partial B_{y, 0} / \partial y\right) .
\end{aligned}
$$

These canonical kicks are the same as the dynamic kicks $\tilde{\theta}_{x}=\Delta x / \Delta z$ and $\tilde{\theta}_{y}=\Delta y / \Delta z$, because the values of the periodical vector potential at the entrance and exit points are the same. It will be shown in Sec. IV that these kicks cannot be derived from 2D magnetic fields. 
The integrated kicks per period of Eq. (39) do agree with results from the kick code, as presented in Eq. (5) of Ref. [11]. However, the applied series expansion Eq. (15) is more general and is developed within the Hamiltonian mechanics. The achieved mapping routine Eq. (27) is valid over a finite step length and it does include the expansion for small initial transverse momenta $p_{x}$ and $p_{y}$, different to [11]. The chosen tracking step length defines the accuracy of approximation, but it is always symplectic. Strong fields require shorter steps, weak fields permit longer steps. For vanishing magnetic fields the long lens turns into a drift of equal length. This mapping routine is comparable to a nonlinear long lens approximation whereas the kick map approximation [11] is based on a sequence of infinitely thin lenses and drifts in between. The speed of the two tracking methods is comparable.

\section{ANALYTIC DESCRIPTION OF THE APPLE II UNDULATOR FIELD}

In the last section, a procedure for an analytic and symplectic particle tracking through arbitrary magnetic fields has been presented. This method is extremely useful for an application in complex 3D undulator magnet fields. The procedure has been illustrated for the example of a planar undulator. The fields of variably polarizing undulators are far more complicated but the procedure is applicable as well if an analytic undulator field model is available. Starting from field descriptions of simple structures an analytic field model for an APPLE II undulator is derived by linear superposition of permanent magnet subunits. The linear superposition is a good approximation since the parallel and perpendicular permeabilities of typical permanent magnet material of $\mu_{\mathrm{par}}=1.04$ and $\mu_{\text {perp }}=1.17$ are small and the fields are overestimated by about $2 \%-3 \%$, only. This field model is used in the tracking algorithm as described in Sec. II.

\section{A. Operation modes of an APPLE II undulator}

An APPLE II magnetic structure consists of four magnet rows (Fig. 1). Horizontal or vertical linearly polarized light as well as elliptically polarized light is produced with a parallel longitudinal motion of two diagonal rows. In this mode the horizontal and vertical field components are always $90^{\circ}$ out of phase and the projection of the trajectory onto the $x-y$ plane is an ellipse with the principle axes parallel to the $x$ and the $y$ axis. This operation mode is called elliptical mode since the emitted light is elliptically polarized. Moving two diagonal rows in opposite directions, the horizontal field and the vertical field are in phase. The trajectory projection is a straight line where the inclination angle to the midplane is determined by the amplitude ratio of the fields. This mode is called inclined mode because now linearly polarized light with an inclination angle to the midplane is emitted. We chose the

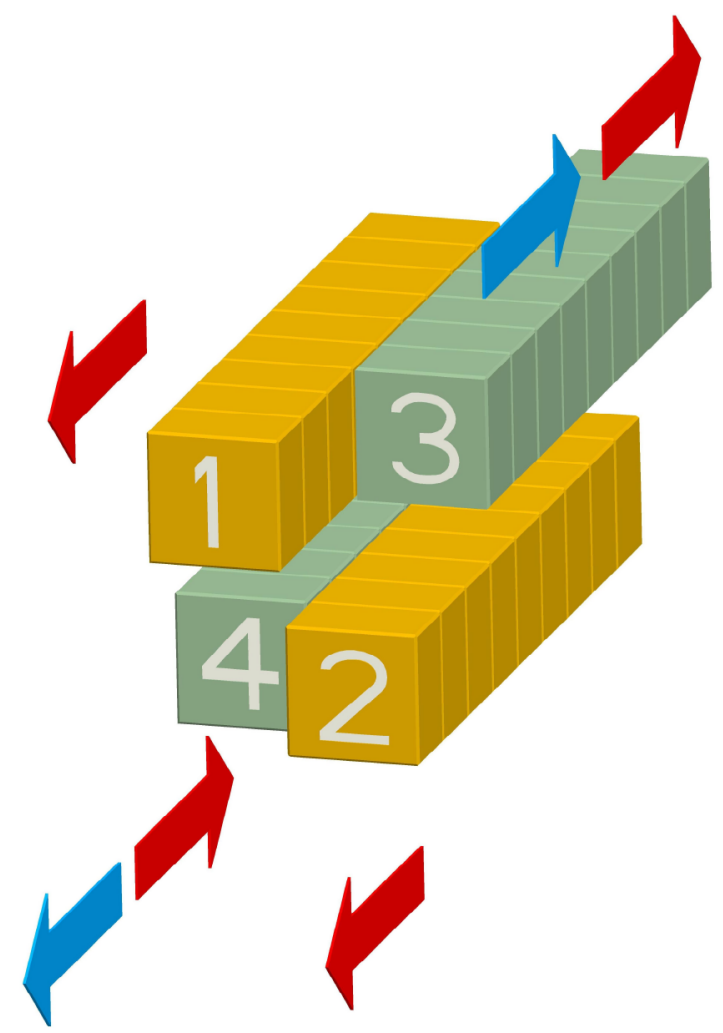

FIG. 1. Operation modes of an APPLE II undulator: In the elliptical mode rows 1 and 2 are moved in one direction $\left(+\varphi_{1} / 2\right)$ and rows 3 and 4 in the opposite direction $\left(-\varphi_{1} / 2\right)$ (red arrows). In the inclined mode row 3 is moved in one direction $\left(-\varphi_{2}\right)$ and row 4 in the opposite direction $\left(+\varphi_{2}\right)$ (blue arrows). In the universal mode both movements are combined. To cover all inclination angles between $0^{\circ}$ and $-90^{\circ}$, row 1 and row 2 are moved in the opposite direction.

operation parameters $\varphi_{1}$ and $\varphi_{2}$ with the following meaning: In the elliptical mode rows 1 and 2 are shifted by $s_{1}=s_{2}= \pm \varphi_{1} / 2 k$ and rows 3 and 4 are shifted by $s_{3}=s_{4}=\mp \varphi_{1} / 2 k$ with $0 \leq \varphi_{1} \leq \pi, k=2 \pi / \lambda_{0}$, and $\lambda_{0}=$ undulator period. Alternatively, rows 1 and 2 could be shifted by $s_{1}=s_{2}= \pm \varphi_{1} / k$ keeping rows 3 and 4 fixed. In the inclined mode row 3 is moved by $s_{3}=$ $-\varphi_{2} / k$ and row 4 by $s_{4}=\varphi_{2} / k$ with $0 \leq \varphi_{2} \leq \pi$.

Beam lines operated in the energy regime below $100 \mathrm{eV}$ suffer from polarization dependent reflectivities, thus, modifying the polarization state of the radiation in a wavelength dependent manner. Contaminations on optical surfaces contribute to polarization changes and may vary in time. In other words, the state of polarization produced by the insertion device is modified by the beam line, e.g., circularly polarized light as produced by the undulator is transformed into light with a tilted polarization ellipse and an amplitude ratio of the principle axes different from one [26]. The characterization of this beam line effect at the experimental station with a polarimeter [27] provides the possibility to precompensate this effect with an appropriate 
setting of the undulator. This is accomplished with an elliptical mode motion by $\varphi_{1}$ and simultaneously an inclined mode motion by $\varphi_{2}$ which we call universal mode (Fig. 1) [26,28]. The movements of the individual rows are given by $s_{1}=s_{2}= \pm \varphi_{1} / 2 k, s_{3}=\left(\mp \varphi_{1} / 2-\varphi_{2}\right) / k$, and $s_{4}=\left(\mp \varphi_{1} / 2+\varphi_{2}\right) / k$.

Various mechanical designs for APPLE II structures have been realized all over the world. Some of them can be operated only in the elliptical mode. In these cases it is sufficient to move only two rows. A stiffer mechanical layout is required in the inclined mode where strong magnetic forces in all three directions show up and the support and drive system has to be capable to handle these forces. The so far largest APPLE II undulator $(5 \mathrm{~m})$ employing the strongest magnetic forces $(70 \mathrm{kN})$ is the PETRA III undulator UE65, which has been built by Helmholtz-Zentrum Berlin [29,30].

Once all four magnet rows can be moved independently, the energy tuning can be done purely by magnet array phasing as done in an adjustable phase undulator [31]. Three degrees of freedom are necessary to adjust the energy and the tilt and the ellipticity of the polarization ellipse. The three independent operation parameters are related to the individual phases of the four rows via

$$
\begin{aligned}
p p & =\left(s_{1}+s_{2}\right) / 2-\left(s_{3}+s_{4}\right) / 2 \\
p a & =\left|s_{1}-s_{2}\right| / 2-\left|s_{3}-s_{4}\right| / 2 \\
p e & =\left(s_{1}+s_{3}\right) / 2-\left(s_{2}+s_{4}\right) / 2 .
\end{aligned}
$$

$p p$ describes the elliptical mode and $p a$ the inclined mode. The third parameter $p e$ is used for an energy tuning if a gap drive is not implemented. At the Swiss Light Source a fixed gap APPLE II undulator has been built providing the full flexibility [32,33].

The universal mode for the production of any arbitrary state of polarization needs also four movable rows (generally, three rows are sufficient, however, more longitudinal space is needed in this case). The BESSY II UE112 has four movable rows but the motions are coupled by software and only two independent parameters are implemented which permit a parallel motion of two diagonal rows and an opposite motion of the other two rows. The energy is adjusted by a gap variation. In Sec. III C we give the recipe to derive the gap and the phases from the energy and the polarization ellipse.

Figure 2 shows the parameter space of an APPLE II undulator with two movable rows. The tilt angle $\psi$ in the inclined mode $\left(0^{\circ}\right.$ phase difference between field components) is limited to $0 \leq \psi \leq \pi / 2$. When switching to the other pair of magnet rows the tuning range is changed to $\pi / 2 \leq \psi \leq \pi$. For any magnetic field amplitude ratio all relative phases between the fields can be achieved (Fig. 2, left). The effective field which determines the photon energy (Fig. 2, right) can be adjusted by choosing an appropriate magnet gap.
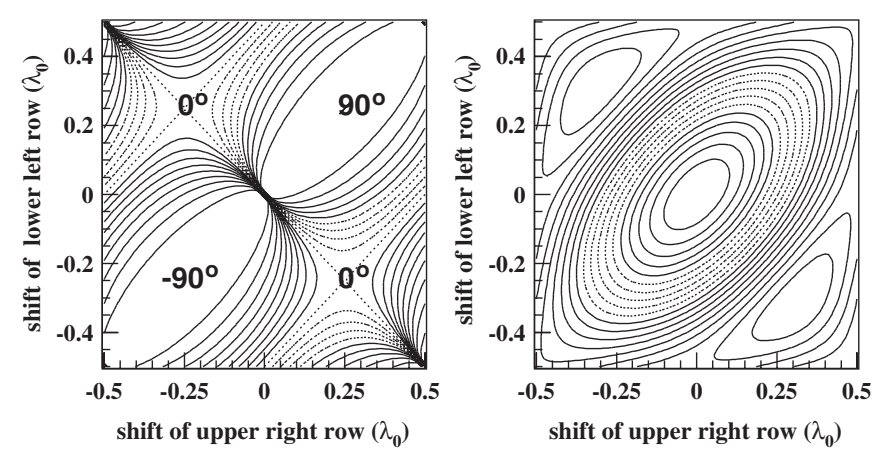

FIG. 2. Parameter space of an APPLE undulator: phase between transverse magnet field components (left) and effective fields (right). The field ratio $B_{y, 0} / B_{x, 0}$ varies between infinity at $s_{1}=s_{2}=0$ and zero at $s_{1}= \pm 0.5$ and simultaneously $s_{2}=$ \pm 0.5 . The data are evaluated for a period length of $56 \mathrm{~mm}$ and a gap of $15 \mathrm{~mm}$ and they change slightly for other parameters.

\section{B. Parametrization of the APPLE II field in three dimensions}

Halbach proposed the following general representation for the fields of a planar vertical field undulator [34]:

$$
\begin{aligned}
B_{x}= & -\frac{B_{0} k_{x}}{k_{y}} \sin \left(k_{x} x\right) \sinh \left(k_{y} y\right) \cos (k z) \\
B_{y}= & B_{0} \cos \left(k_{x} x\right) \cosh \left(k_{y} y\right) \cos (k z) \\
B_{z}= & -\frac{B_{0} k}{k_{y}} \cos \left(k_{x} x\right) \sinh \left(k_{y} y\right) \sin (k z) \\
& -k_{x}^{2}+k_{y}^{2}=k^{2}=\left(2 \pi / \lambda_{0}\right)^{2} .
\end{aligned}
$$

The parameters $k_{x}, k_{y}$, and $k$ are related via Maxwell's equations (last line). $k_{x}$ can be either real or imaginary, representing a horizontally defocusing or focusing device, respectively. Similar expressions can be found for a $90^{\circ}$ rotated planar device producing horizontal fields. In principle, helical or inclined linear on-axis fields can be constructed via a linear superposition of these fields. However, the complicated off-axis field distribution which causes the influence on the electron beam dynamics is difficult to describe in such an approach. Furthermore, the specific on-axis field dependence on the magnet row movement in an APPLE undulator cannot be described.

In the following we will present an extended model which is well adapted to the specific design of an APPLE II type undulator and easily applicable to other magnet designs. A parametrization of the fields of one individual magnet row permits the extrapolation of the fields of the complete undulator by linear superposition of the contributions from all four rows. The parametrization of one magnet row follows the ansatz of Halbach with an additional term accounting for the gap dependency [Eq. (42)]. The functions on the right-hand side of Eq. (42) form a complete and orthogonal basis for all functions $\left(B_{x}, B_{y}, B_{z}\right)$ which are periodic in the $x-z$ plane $\left(B_{y}\right.$ symmetric in $x$ ) and which obey the 3D Maxwell 
equations. The row is symmetric in $x$ and it is located below the midplane. The field at $y=0$ corresponds to a given distance to the magnet surface of $g_{0} / 2$, where $g_{0}$ is the vertical distance between the upper and lower magnet row, the so-called magnet gap of the complete device. $\Delta g$ is the deviation from the nominal gap of $g_{0}$ :

$$
\begin{aligned}
B_{x}= & \sum_{i=0}^{n} \sum_{j=1}^{m} \frac{k_{x i}}{k_{y i, \tilde{j}}} c_{i, \tilde{j}} \sin \left(k_{x i} x\right) \exp \left(-k_{y i, \tilde{j}} y\right) \cos \left(k_{\tilde{j}} z+\varphi\right) \\
& \times \exp \left(-k_{y i, \tilde{j}} \Delta g / 2\right) \\
B_{y}= & \sum_{i=0}^{n} \sum_{j=1}^{m} c_{i, \tilde{j}} \cos \left(k_{x i} x\right) \exp \left(-k_{y i, \tilde{j}} y\right) \cos \left(k_{\tilde{j}} z+\varphi\right) \\
& \times \exp \left(-k_{y i, \tilde{j}} \Delta g / 2\right) \\
B_{z}= & \sum_{i=0}^{n} \sum_{j=1}^{m} \frac{k}{k_{y i, \tilde{j}}} c_{i, \tilde{j}} \cos \left(k_{x i} x\right) \exp \left(-k_{y i, \tilde{j}} y\right) \cos \left(k_{\tilde{j}} z+\varphi\right) \\
& \times \exp \left(-k_{y i, \tilde{j}} \Delta g / 2\right) \\
\tilde{j}= & 1+l(j-1) \quad k_{x i}=k_{x 1} i=\left(2 \pi / \lambda_{x 0}\right) i \\
k_{y i, \tilde{j}}= & \sqrt{k_{\tilde{j}}^{2}+k_{x i}^{2}} \quad k_{\tilde{j}}=k \cdot \tilde{j} .
\end{aligned}
$$

For a list of abbreviations see Appendix B. These expressions assume an easy axis rotation between subsequent magnets of $360^{\circ} / l$ ( $l$ magnets of identical shape per period). It is worth noting that, in a pure permanent magnet undulator with four blocks of identical shape per period and under the assumption $\mu=1$, only the harmonics 1,5 , $9, \ldots$ show up in the longitudinal field distribution [34]. Otherwise, all odd harmonics are present $(1,3,5, \ldots)$. Equation (42) describes magnet blocks with a symmetric shape in transverse direction. If this is not the case (e.g. the magnet blocks have cut outs for clamping) additional terms have to be added (see scalar potential in Appendix C). Equation (42) is a Fourier series expansion of the field of one individual magnet row with respect to transverse and longitudinal coordinates. $\lambda_{x 0}$ is the range for the transverse Fourier decomposition. The Fourier coefficients $c_{i, \tilde{j}}$ are determined as follows: The transverse vertical field distribution of a single row at the $z$ position of maximum field and the nominal vertical distance of $g_{0} / 2$ is evaluated numerically (e.g. with RADIA $[35,36]$ ) and decomposed into a Fourier series with coefficients $C_{i, 1}$. The same procedure is applied to field distributions at other distances $g_{0} / 2+\Delta y_{j}$ yielding $m$ sets of coefficients $C_{i, j}$. Using Eqs. (42) and (43) a set of $(n+1) \cdot m$ linear equations correlating the constants $C_{i, j}$ and the unknowns $c_{i, \tilde{j}}$ is set up and solved for the coefficients $c_{i, \tilde{j}}$ :

$$
\begin{aligned}
& C_{0,1}=\sum_{j=1}^{m} c_{0, \tilde{j}} \\
& \ldots \\
& C_{n, 1}=\sum_{j=1}^{m} c_{n, \tilde{j}} \\
& \ldots \\
& C_{n, m}=\sum_{j=1}^{m} c_{n, \tilde{j}} \exp \left(-\sqrt{k^{2} \tilde{j}^{2}+k_{x n}^{2}} \Delta y_{m}\right) .
\end{aligned}
$$

$m$ defines the accuracy in the $y-z$ plane and $n$ the accuracy in $x$.

Based on the field expressions of Eq. (42), the complete APPLE II undulator field is derived via an appropriate transverse positioning and longitudinal phasing of the four individual rows. The scalar potential of a complete APPLE II structure is given in Appendix C. The fields as determined by derivation are given in Appendix D. The gap difference to the nominal gap $g_{0}$ is included with $\Delta g$. The fields have the following phase dependence: In the elliptical mode $\left(\varphi_{2}=0\right)$ each transverse field component consists of two terms proportional to $\cos \left(\varphi_{1} / 2\right)$ and $\sin \left(\varphi_{1} / 2\right)$. In the inclined mode $\left(\varphi_{1}=0\right)$ the field components are sums of three terms proportional to $\cos ^{2}\left(\varphi_{2} / 2\right)$, $\sin ^{2}\left(\varphi_{2} / 2\right)$, and $\cos \left(\varphi_{2} / 2\right) \sin \left(\varphi_{2} / 2\right)$.

In Sec. II analytic expressions of the vector potential are used for particle tracking through undulators. The vector potential is related to the scalar potential via Eq. (28). The input parameters for the tracking are the gap and the phases of the magnet rows and a table of typically 30 Fourier coefficients describing the main undulator field. These coefficients are evaluated numerically. A single table of coefficients is sufficient to simulate all operation modes, i.e., all phases and all gaps of the undulator. If needed, a second table with the same number of Fourier coefficients describing correction magnets (see Sec. V) is provided. Thus, the tracking studies are very flexible and can be performed over a large parameter space without repetitive numeric field simulations.

\section{On-axis field of an APPLE II undulator}

The spectral performance of an undulator is defined by the on-axis fields as derived from Appendix D:

$$
\begin{aligned}
B_{x} & =\sum_{i=0}^{n} \sum_{j=1}^{m} 4 c_{i, \tilde{j}} \frac{k_{x i}}{k_{y i, \tilde{j}}} e_{i, \tilde{j}}\left(-s_{x i 0} c_{z \tilde{j}} c_{p p} s_{p a}^{2}-s_{x i 0} s_{z \tilde{j}} s_{p p} c_{p a}^{2}\right) \quad B_{y}=\sum_{i=0}^{n} \sum_{j=1}^{m} 4 c_{i, \tilde{j}} e_{i, \tilde{j}}\left(c_{x i 0} c_{z j} c_{p p} c_{p a}^{2}+c_{x i 0} s_{z j} s_{p p} s_{p a}^{2}\right) \\
B_{z} & =\sum_{i=0}^{n} \sum_{j=1}^{m} 4 c_{i, \tilde{j}} \frac{k}{k_{y i, \tilde{j}}} e_{i, \tilde{j}}\left(-c_{x i 0} c_{z \tilde{j}} c_{p p} c_{p a} s_{p a}+c_{x i 0} s_{z \tilde{j}} s_{p p} c_{p a} s_{p a}\right) .
\end{aligned}
$$


The abbreviations are defined in Appendix B. The accuracy of the Fourier decomposition with $n=60$ and various values of $m$ is demonstrated in Fig. 3 which gives the results for the BESSY UE112 APPLE II undulator. Even $8 \mathrm{~mm}$ off axis within a $20 \mathrm{~mm}$ gap the field errors are below 3\%. With this set of coefficients we have an accurate and fully parametrized model for all gaps and all operation modes such as the helical, inclined, and universal mode. We remind that this model is based on the linear superposition of the fields from the four rows.

The user of a synchrotron radiation light source specifies the photon energy $E$ and a state of polarization as defined by the normalized Stokes parameters $S_{1}, S_{2}$, and $S_{3}$. These parameters, $E, S_{1}, S_{2}$, and $S_{3}$, have to be translated into the undulator settings. Assuming a degree of polarization of $100 \%$ the Stokes parameters have to satisfy $S_{1}^{2}+S_{2}^{2}+$ $S_{3}^{2}=1$ and, thus, we have three independent parameters. The electron trajectory has to be adjusted according to the state of polarization. The polarization ellipse is defined by two parameters, the angle of inclination and the ellipticity. The third parameter is the undulator gap which has to be adjusted for the chosen energy. The inclination of the polarization ellipse $\psi$ is related to the Stokes parameters and the ratio of the vertical and horizontal electric field component via

$$
\frac{E_{y 0}}{E_{x 0}}=\tan (\psi)=\tan \left(0.5 \arctan \frac{S_{2}}{S_{1}}\right) .
$$

The ellipticity of the polarization ellipse is defined by the phase relation $\delta=\delta_{y}-\delta_{x}$ between the two orthogonal electric field components which is related to the Stokes parameters via

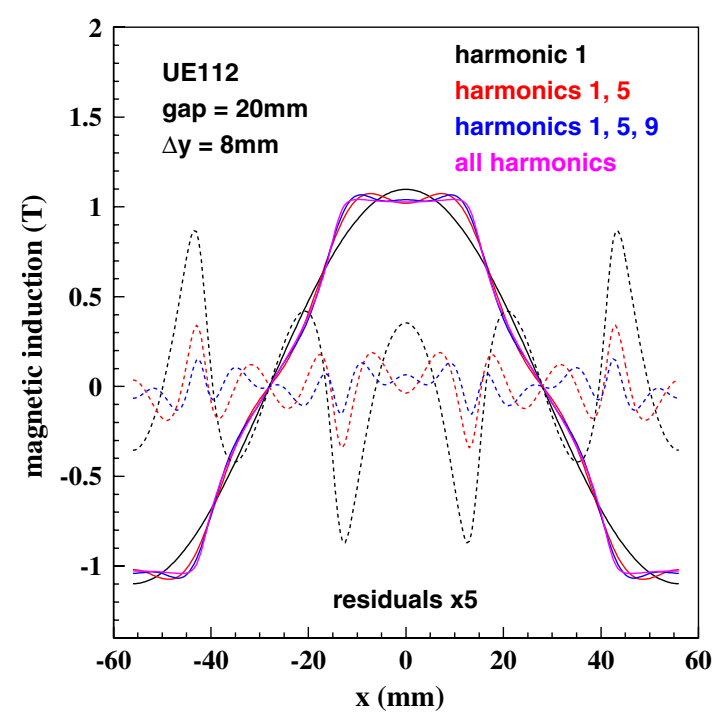

FIG. 3. Accuracy of field prediction from analytic model at large vertical offsets depending on the number of Fourier components used. The magenta curve corresponds to numeric simulations.

$$
\delta=\arctan \frac{S_{3}}{S_{2}} .
$$

The ratio of the electric field components and their phase difference defines the required electron trajectory in terms of the ratio of the two orthogonal magnetic field components and the phase relation between them. The third parameter is the effective magnetic field which defines the photon energy. These three parameters, $B_{y, 00} / B_{x, 00}=$ $B_{y, 0}(x=0, y=0) / B_{x, 0}(x=0, y=0), \quad \delta$, and $B_{\mathrm{eff}, 00}=$ $B_{\text {eff }, 0}(x=0, y=0)$, are translated into the undulator operation parameters $\varphi_{1}, \varphi_{2}$, and $\Delta g$.

The user is only interested in photon energies. These are related to the path length of the electrons within one undulator period. The path length is evaluated from the effective fields. The effective vertical and horizontal fields are given by quadratic sums over the longitudinal Fourier components and the total effective field is a quadratic sum of the transverse components:

$$
B_{\mathrm{eff}, 00}=\sqrt{\sum_{j=1}^{m} \frac{B_{x, \tilde{j}, 00}^{2}+B_{y, \tilde{j}, 00}^{2}}{\tilde{j}^{2}}},
$$

where $B_{x, \tilde{j}, 00}, B_{y, \tilde{j}, 00}$ are the maxima of the $\tilde{j}$ th longitudinal Fourier component of the transverse fields. The higher field harmonics contribute only weakly to the effective field $B_{\text {eff,00 }}$ since (i) they are added quadratically and (ii) they are suppressed with the factor $1 / \tilde{j}^{2}$. Therefore, in the following we will discuss only the first longitudinal harmonic. Nevertheless, a Fourier decomposition with respect to the longitudinal coordinate has to be done beforehand in order to determine the coefficients of the first harmonic. The field equations can be rewritten in the general form:

$$
\begin{aligned}
B_{x} & =D(\Delta g)\left(c_{p p} s_{p a}^{2} c_{z}+s_{p p} c_{p a}^{2} s_{z}\right) \\
& =B_{x, 00}\left(\varphi_{1}, \varphi_{2}, \Delta g\right) \cos \left[k z+\delta_{x}\left(\varphi_{1}, \varphi_{2}\right)\right] \\
B_{y} & =E(\Delta g)\left(c_{p p} c_{p a}^{2} c_{z}+s_{p p} s_{p a}^{2} s_{z}\right) \\
& =B_{y, 00}\left(\varphi_{1}, \varphi_{2}, \Delta g\right) \cos \left[k z+\delta_{y}\left(\varphi_{1}, \varphi_{2}\right)\right] \\
B_{x, 00}^{2} & =D^{2}\left[\left(c_{p p} s_{p a}^{2}\right)^{2}+\left(s_{p p} c_{p a}^{2}\right)^{2}\right] \\
B_{y, 00}^{2} & =E^{2}\left[\left(c_{p p} c_{p a}^{2}\right)^{2}+\left(s_{p p} s_{p a}^{2}\right)^{2}\right] \\
D & =-\sum_{i=0}^{n} 4 c_{i} s_{x i 0} \frac{k_{x i}}{k_{y i}} e_{i} \quad E=\sum_{i=0}^{n} 4 c_{i} c_{x i 0} e_{i} \\
\delta_{x} & =\arctan \left[-\left(s_{p p} c_{p a}^{2}\right) /\left(c_{p p} s_{p a}^{2}\right)\right] \\
\delta_{y} & =\arctan \left[-\left(s_{p p} s_{p a}^{2}\right) /\left(c_{p p} c_{p a}^{2}\right)\right] .
\end{aligned}
$$

The operation parameters $\varphi_{1}, \varphi_{2}$, and $\Delta g$ required to produce a specified photon energy and state of polarization are evaluated as follows: 


$$
\begin{aligned}
\cos ^{2}\left(\varphi_{2} / 2\right) & =\frac{1}{2} \pm \frac{1}{2} \sqrt{\frac{2 B_{\mathrm{eff}, 00}^{2}(P+Q)}{\left(E^{2} P+D^{2} Q\right)}-1} \\
\cos ^{2}\left(\varphi_{1} / 2\right) & =\frac{-P \cos ^{4}\left(\varphi_{2} / 2\right)+Q\left[1-\cos ^{2}\left(\varphi_{2} / 2\right)\right]^{2}}{\left[1-2 \cos ^{2}\left(\varphi_{2} / 2\right)\right](P+Q)} \\
P & =\left(B_{y, 00} / B_{x, 00}\right)^{2} \quad Q=(E / D)^{2} .
\end{aligned}
$$

These expressions of $\varphi_{1}$ and $\varphi_{2}$ are inserted into Eq. (50) which is an implicit equation of the third parameter $\Delta g$ :

$$
\begin{aligned}
f(\Delta g)= & \arctan \left[\tan \left(\varphi_{1} / 2\right) \tan ^{2}\left(\varphi_{2} / 2\right)\right] \\
& -\arctan \left[\tan \left(\varphi_{1} / 2\right) / \tan ^{2}\left(\varphi_{2} / 2\right)\right]-\delta=0 \\
\delta= & \delta_{y}-\delta_{x} .
\end{aligned}
$$

$\Delta g$ is evaluated iteratively from Eq. (50) using the Newton Raphson method. The algorithm makes use of the first derivative of $f(\Delta g)$ with respect to $\Delta g$ which is evaluated from the analytic form of Eqs. (49) and (50) with an algebraic code like REDUCE [25]. The derivatives of $D$ and $E$ with respect to $\Delta g$ are given by

$$
\frac{\partial D}{\partial \Delta g}=\sum_{i=0}^{n} 2 c_{i} s_{x i 0} k_{x i} e_{i} \quad \frac{\partial E}{\partial \Delta g}=-\sum_{i=0}^{n} 2 c_{i} c_{x i 0} k_{y i} e_{i} .
$$

In the elliptical mode the phase $\varphi_{1}$ required to produce an amplitude ratio of $\sqrt{P}=\left|B_{y, 00} / B_{x, 00}\right|$ is evaluated from

$$
\varphi_{1}=2 \arccos \sqrt{\frac{P}{P+Q}} .
$$

In the inclined mode the phase $\varphi_{2}$ for the production of a tilt angle between the wiggling plane and the midplane of $\psi=\arctan \left(B_{y, 00} / B_{x, 00}\right)$ is given by Eq. (53). The equation includes two solutions. Only one of them is real and represents the desired parameter:

$$
\varphi_{2}=2 \arccos \sqrt{\frac{-P}{P-Q} \pm \sqrt{\frac{P Q}{(P-Q)^{2}}}} .
$$

The phases in Eqs. (52) and (53) are gap dependent. This dependency is included in the terms $P$ and $Q$ which include $\Delta g$ in the exponential factors.

\section{ANALYTIC MODEL OF DYNAMIC FIELD INTEGRALS}

The analytic field description of the last section permits an analytic particle tracking based on the generating function algorithm of Sec. II. For tune feed forward schemes and for the use in active shimming concepts (they will be discussed in Sec. V), analytic expressions are needed for the evaluation of dynamic field integrals which are related to the wiggling motion of particles in an undulator. Static field integrals are defined as straight line integrals which can be measured with a wire or similar equipment. In contrast, the straight line integrals of 2 nd order kicks are zero. Their effect to the beam is represented by so-called dynamic field integrals. These dynamic field integrals do not fulfill the 2D-Maxwell equations and even on a circular disk within the free aperture they cannot be described with a two-dimensional multipole expansion as it is usually done for accelerator magnets. In this section we derive analytic expressions of dynamic field integrals in two dimensions and expressions for tune shifts and coupling terms between planes. The discussion will concentrate on APPLE II structures but it can be applied easily also to other designs.

\section{A. Definition of dynamic field integrals}

Dynamic field integrals can be observed in the fringe fields of dipole magnets. Undulators or wigglers which are basically a sequence of alternating dipole magnets have a strong influence on the electron beam dynamic. In Sec. IID 2 the dynamic kicks $\tilde{\theta}_{x}$ and $\tilde{\theta}_{y}$ of a particle moving through a planar undulator, represented by one longitudinal and one transverse Fourier component, are derived [Eq. (39)]. For a realistic model of an undulator more Fourier components in transverse and longitudinal direction have to be included (Sec. III). Further terms have to be added in complex structures such as an APPLE II undulator. The validity of linear superposition of the vector potential terms justifies a simple summation over all contributions. The complete expressions of the dynamic kicks of an APPLE II undulator operated in the universal mode are given in Appendix E. The contributions from the field harmonics in longitudinal direction scale with $1 / k_{i}^{2}=$ $\left(\lambda_{0} / i \cdot 2 \pi\right)^{2}$, where $i$ is the order of the harmonic (only odd harmonics appear). Thus, close to the midplane higher longitudinal harmonics contribute only little to the kicks and can usually be neglected.

We define the dynamic field integrals $\tilde{B}_{x}$ and $\tilde{B}_{y}$ (in Tmm) such that straight line integrals with equal values produce kicks $\bar{\theta}_{x}$ and $\bar{\theta}_{y}$ with the same strength as the dynamic kicks $\tilde{\theta}_{x}$ and $\tilde{\theta}_{y}$. From the Lorenz equation $\vec{F} \propto \vec{v} \times \vec{B} \propto\left(\tilde{B}_{y},-\tilde{B}_{x}\right)$ we find the relation between the dynamic field integrals and the dynamic kicks:

$$
f_{002 x}=\tilde{\theta}_{x}=\tilde{B}_{y} / B \rho \quad f_{002 y}=\tilde{\theta}_{y}=-\tilde{B}_{x} / B \rho .
$$

\section{B. Comparison of static and dynamic multipoles}

The discussion of this section is limited to the region of a circular disk within the free aperture in the undulator gap. It is well known that two-dimensional (2D) real multipoles (multipoles of a 2D distribution of straight line field integrals) obey the 2D-Maxwell equations. In other words, an analytic function $\bar{F}=\bar{B}_{x}-i \bar{B}_{y}$ can be constructed from the static field integrals $\bar{B}_{x / y}=\int_{-\infty}^{\infty} B_{x / y} d z$. The Cauchy Riemann relations of 


$$
\frac{\partial \bar{B}_{x}}{\partial x}=\frac{\partial\left(-\bar{B}_{y}\right)}{\partial y} \quad-\frac{\partial\left(-\bar{B}_{y}\right)}{\partial x}=\frac{\partial \bar{B}_{x}}{\partial y}
$$

are another formulation of Maxwell's equations $\vec{\nabla} \times \overrightarrow{\bar{B}}=0$ and $\vec{\nabla} \cdot \overrightarrow{\bar{B}}=0$ with $\overrightarrow{\bar{B}}=\left(\bar{B}_{x}, \bar{B}_{y}\right)$. In contrast, the complex function $\tilde{F}=\tilde{B}_{x}-i \tilde{B}_{y}$ is not an analytic function.

We examine the divergence and the rotation of the dynamic field integrals. The kick of a particle passing through an undulator is given in Eq. (39) as

$$
\Delta p_{x}=f_{002 x} \quad \Delta p_{y}=f_{002 y} .
$$

These dynamic kicks are related to dynamic field integrals via Eq. (54). With Eq. (54) the Maxwellian character of these terms can be checked. The divergence of the dynamic field integrals is given as

$$
\begin{aligned}
\vec{\nabla} \cdot\left(\tilde{B}_{x}, \tilde{B}_{y}\right) & =\partial \tilde{B}_{x} / \partial x+\partial \tilde{B}_{y} / \partial y \propto-f_{002 y x}+f_{002 x y} \\
& =0
\end{aligned}
$$

and the rotation is

$$
\begin{aligned}
{\left[\vec{\nabla} \times\left(\tilde{B}_{x}, \tilde{B}_{y}\right)\right]_{z} } & =\partial \tilde{B}_{y} / \partial x-\partial \tilde{B}_{x} / \partial y \propto f_{002 x x}+f_{002 y y} \\
& \neq 0 .
\end{aligned}
$$

These expressions show that the divergence of dynamic field integrals is always zero whereas the rotation is generally nonzero. To stress this difference we call the expansion terms of the transverse distribution of dynamic field integrals "dynamic multipoles" as opposed to static multipoles. Static and dynamic multipoles represent different sets of functions and, therefore, it is impossible to compensate dynamic multipoles with shims which are represented by static multipoles.

For an illustration of the dynamic field integrals we examine a planar undulator. We consider only one Fourier component and we restrict the discussions to the relevant dynamic multipoles of an ideal undulator. These are regular dynamic quadrupoles, octupoles, dodecapoles, etc. We use similar names as for the static multipoles to indicate identical exponents of the transverse coordinates $x$ and $y$ though the prefactors behave differently from static multipoles. The field integrals of the transverse field components of the static regular multipoles are described by

$$
\begin{aligned}
\bar{B}_{y}^{(n)} & =\sum_{i=1}^{\lfloor n / 2\rfloor}(-1)^{i-1} \bar{a}_{y n i} \cdot x^{n+1-2 i} y^{2(i-1)} \\
\bar{B}_{x}^{(n)} & =\sum_{i=1}^{\lfloor n / 2\rfloor}(-1)^{i-1} \bar{a}_{x n i} \cdot x^{n-2 i} y^{2(i-1)+1},
\end{aligned}
$$

where $n$ is the order of multipole $(n=1,2,3, \ldots$ means dipole, quadrupole, sextupole, ...). All prefactors $\bar{a}_{x n i}, \bar{a}_{y n i}$ have the same sign for a given $n$. In an ideal undulator we have $\bar{B}_{y}^{(n)}=0, \forall n$.
The dynamic multipoles of a planar undulator with one longitudinal and one transverse Fourier harmonic are derived from Eq. (39). As usual, we have $k_{x}^{2}>0$ for a horizontal defocusing and $k_{x}^{2}<0$ for a horizontal focusing undulator:

$$
\begin{aligned}
\tilde{B}_{y}^{(n)}= & -\delta_{0, \bmod (n, 2)} \frac{L}{8 B \rho k^{2}}\left[-\left(\frac{k_{x}^{3}}{k_{y}^{2}}+k_{x}\right)(-1)^{n / 2-1}\right. \\
& \times \frac{1}{(n-1) !}\left(2 k_{x}\right)^{n-1} x^{n-1}+\left(\frac{k_{x}^{3}}{k_{y}^{2}}-k_{x}\right) \sum_{i=1}^{n / 2}(-1)^{i+1} \\
& \left.\times \frac{1}{(2 i-1) !} \frac{1}{(n-2 i) !}\left(2 k_{x}\right)^{2 i-1}\left(2 k_{y}\right)^{n-2 i} x^{2 i-1} y^{n-2 i}\right],
\end{aligned}
$$

$$
\begin{aligned}
\tilde{B}_{x}^{(n)}= & \delta_{0, \bmod (n, 2)} \frac{L}{8 B \rho k^{2}}\left[-\frac{k_{x}^{2}}{k_{y}^{2}} k_{y} \sum_{i=1}^{n / 2}(-1)^{i+1} \frac{1}{(2 i-2) !}\right. \\
& \times\left(2 k_{x}\right)^{2 i-2} x^{2 i-2} \frac{1}{(n-2 i+1) !}\left(2 k_{y}\right)^{n-2 i+1} y^{n-2 i+1} \\
& +k_{y} \sum_{i=1}^{n / 2}(-1)^{i+1} \frac{1}{(2 i-2) !}\left(2 k_{x}\right)^{2 i-2} x^{2 i-2} \\
& \times \frac{1}{(n+1-2 i) !}\left(2 k_{y}\right)^{n+1-2 i} y^{n+1-2 i} \\
& \left.+\left(\frac{k_{x}^{2}}{k_{y}^{2}} k_{y}+k_{y}\right) \frac{1}{(n-1) !}\left(2 k_{y}\right)^{n-1} y^{n-1}\right]
\end{aligned}
$$

In the following we will consider three limiting cases: (1) The undulator focuses horizontally with $-k_{x}^{2}=k_{y}^{2}=$ $k^{2} / 2$ which requires a specific pole shaping in planar undulators. With $\tilde{a}_{y n i}, \tilde{a}_{x n i}>0, \forall n$ we have

$$
\begin{aligned}
& \tilde{B}_{y}^{(n)} \propto-\sum_{i=1}^{n / 2} \tilde{a}_{y n i} x^{n+1-2 i} y^{2(i-1)} \\
& \tilde{B}_{x}^{(n)} \propto \sum_{i=1}^{n / 2} \tilde{a}_{x n i} x^{n-2 i} y^{2(i-1)+1}
\end{aligned}
$$

(2) The undulator defocuses horizontally and the period length is long as compared to transverse field profile: $k_{x}^{2} \approx k_{y}^{2}$. With $\tilde{b}_{y n}, \tilde{b}_{x n}>0, \forall n$ the dynamic field integrals are

$$
\tilde{B}_{y}^{(n)} \propto \tilde{b}_{y n}(-1)^{n / 2-1} x^{n-1} \quad \tilde{B}_{x}^{(n)} \propto \tilde{b}_{x n} y^{n-1} .
$$

(3) The undulator poles are assumed to be infinitely wide: $k_{x}^{2}=0$. With $\tilde{c}_{x n}>0, \forall n$ we have

$$
\tilde{B}_{y}^{(n)} \propto 0 \quad \tilde{B}_{x}^{(n)} \propto \tilde{c}_{x n} y^{n-1} .
$$

The prefactors $\tilde{a}_{y n i}, \tilde{a}_{x n i}, \tilde{b}_{y n}, \tilde{b}_{x n}, \tilde{c}_{n x}$ follow from Eqs. (60) and (61).

In the first case all terms of the static multipoles [Eq. (59)] show up also in the dynamic multipoles 
[Eq. (62)]. However, the signs are different. In the second case the cross terms of the static multipoles do not show up in the dynamic multipoles [Eq. (63)] and the remaining terms appear with different signs. In the third case one component is always zero [Eq. (64)] which is never the case for static multipoles. These differences illustrate the non-Maxwellian character of the dynamic multipoles.

\section{Dynamic field integrals in the midplane}

For the elliptical and the inclined mode, it has been demonstrated numerically [37] that for a specific gap and arbitrary row phases the dynamic field integrals in the midplane can be represented with a few generic functions. In the elliptical case two generic functions, $f_{0}$ and $f_{\pi}$, describe the horizontal kicks whereas the vertical kicks are zero. In the inclined mode three generic functions, $f_{0}, f_{\pi}$, and $f_{\pi / 2}$, describe the horizontal kicks and another function, $g_{\pi / 2}$, the vertical kicks. Based on the field expressions given in Appendix D analytic expressions of the dynamic field integrals in two dimensions are derived using Eq. (39). General expressions for the universal mode are given in Appendix E. The following four generic functions are special cases of the general equations: elliptical mode $\left(\varphi_{2}=0\right)$ :

$$
\begin{aligned}
c_{p a} & =1 \quad s_{p a}=0 \quad \theta_{x}=f_{0} c_{p p}^{2}+f_{\pi} s_{p p}^{2} \\
\theta_{y} & =0 \quad f_{0}=\frac{L 8}{k^{2}(B \rho)^{2}} \sum_{i=0}^{n} \sum_{j=0}^{n} c_{i} c_{j} e_{i} e_{j} k_{x j} c_{x i 0} c_{x i} c_{x j 0} s_{x j} \\
f_{\pi} & =\frac{L 8}{k^{2}(B \rho)^{2}} \sum_{i=0}^{n} \sum_{j=0}^{n} c_{i} c_{j} e_{i} e_{j} \frac{k_{x i}}{k_{y i}} \frac{k_{x j}^{2}}{k_{y j}} s_{x i 0} c_{x i} s_{x j 0} s_{x j} ;
\end{aligned}
$$

inclined mode $\left(\varphi_{1}=0\right)$ :

$$
\begin{aligned}
c_{p p}= & 1 \quad s_{p p}=0 \quad \theta_{x}=f_{0} c_{p a}^{4}+f_{\pi} s_{p a}^{4}+f_{\pi / 2} c_{p a}^{2} s_{p a}^{2} \\
\theta_{y}= & g_{\pi / 2} c_{p a}^{2} s_{p a}^{2} \\
f_{\pi / 2}= & -\frac{L 8}{k^{2}(B \rho)^{2}} \sum_{i=0}^{n} \sum_{j=0}^{n} c_{i} c_{j} e_{i} e_{j} \\
& \times\left(\frac{k_{x i}}{k_{y i}} \frac{k_{x j}^{2}}{k_{y j}} c_{x i 0} s_{x i} c_{x j 0} c_{x j}+k_{x j} s_{x i 0} s_{x i} s_{x j 0} c_{x j}\right) \\
g_{\pi / 2}= & -\frac{L 8}{k^{2}(B \rho)^{2}} \sum_{i=0}^{n} \sum_{j=0}^{n} c_{i} c_{j} e_{i} e_{j} \\
& \times\left(\frac{k_{x i}}{k_{y i}} k_{x j}\left(s_{x i 0} c_{x i} c_{x j 0} s_{x j}-c_{x i 0} s_{x i} s_{x j 0} c_{x j}\right)\right. \\
& \left.+k_{y j}\left(-c_{x i 0} c_{x i} s_{x j 0} s_{x j}+s_{x i 0} s_{x i} c_{x j 0} c_{x j}\right)\right) .
\end{aligned}
$$

The shape of the generic functions is gap dependent since the higher order Fourier terms decrease faster with
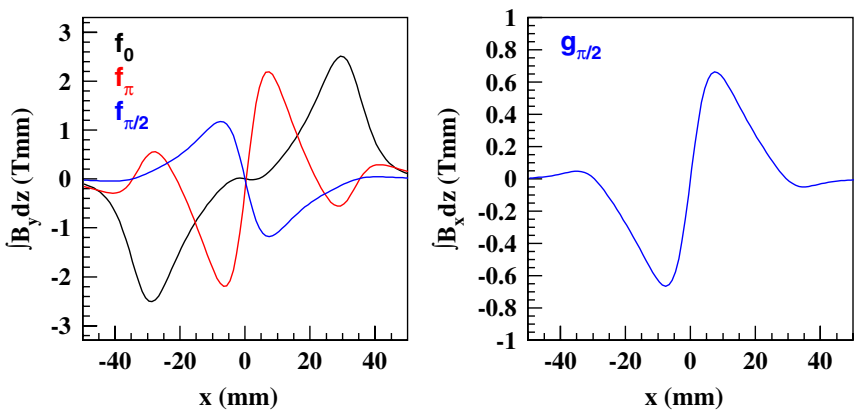

FIG. 4. Generic functions $f_{0}, f_{\pi}, f_{\pi / 2}, g_{\pi / 2}$ for the description of the dynamic multipoles in the elliptic and inclined mode. The values are given for the BESSY II UE112 at smallest gap of $20 \mathrm{~mm}$ in the midplane.

gap than the lower order terms. The generic functions of the UE112 at gap $=20 \mathrm{~mm}$ are plotted in Fig. 4. This representation can be used for an online evaluation of the dynamic field integrals in an active compensation scheme (see Sec. V).

\section{Linear terms in the midplane}

The partial derivatives of the dynamic field integrals are related to the horizontal and vertical tune shifts via

$$
\Delta Q_{x}=-\frac{1}{4 \pi} \frac{\partial \theta_{x}}{\partial x}\left\langle\beta_{x}\right\rangle_{L} \quad \Delta Q_{y}=\frac{1}{4 \pi} \frac{\partial \theta_{y}}{\partial y}\left\langle\beta_{y}\right\rangle_{L},
$$

where the average is taken over the undulator length.

The kicks for the universal mode are given in Appendix $\mathrm{E}$ and the evaluation of the derivatives is straightforward. In the elliptical mode the derivatives of the dynamic kicks on axis are

$$
\begin{aligned}
\partial \theta_{x} / \partial x= & -\frac{8 L}{(B \rho)^{2} k^{2}} \sum_{i=0}^{n} \sum_{j=0}^{n} c_{i} c_{j} e_{i} e_{j} \\
& \times\left(-\frac{k_{x i}}{k_{y i}} \frac{k_{x j}^{3}}{k_{y j}} s_{x i 0} s_{x j 0} s_{p p}^{2}-k_{x j}^{2} c_{x i 0} c_{x j 0} c_{p p}^{2}\right) \\
\partial \theta_{y} / \partial y= & -\frac{8 L}{(B \rho)^{2} k^{2}} \sum_{i=0}^{n} \sum_{j=0}^{n} c_{i} c_{j} e_{i} e_{j} \\
& \times\left(\frac{k_{x i}}{k_{y i}} k_{x j} k_{y j} s_{x i 0} s_{x j 0} s_{p p}^{2}+k_{y j}^{2} c_{x i 0} c_{x j 0} c_{p p}^{2}\right) \\
\partial \theta_{x} / \partial y= & \partial \theta_{y} / \partial x=0 .
\end{aligned}
$$

The coupling terms are zero and, thus, the planes are decoupled. The kick derivatives in the inclined mode on axis are 


$$
\begin{aligned}
\partial \theta_{x} / \partial x= & -\frac{8 L}{(B \rho)^{2} k^{2}} \sum_{i=0}^{n} \sum_{j=0}^{n} c_{i} c_{j} e_{i} e_{j} \\
& \times\left[\left(\frac{k_{x i}^{2}}{k_{y i}} \frac{k_{x j}^{2}}{k_{y j}} c_{x i 0} c_{x j 0}+k_{x i} k_{x j} s_{x i 0} s_{x j 0}\right) c_{p a}^{2} s_{p a}^{2}\right. \\
& \left.-\frac{k_{x i}}{k_{y i}} \frac{k_{x j}^{3}}{k_{y j}} s_{x i 0} s_{x j 0} s_{p a}^{4}-k_{x j}^{2} c_{x i 0} c_{x j 0} c_{p a}^{4}\right] \\
\partial \theta_{y} / \partial y= & -\frac{8 L}{(B \rho)^{2} k^{2}} \sum_{i=0}^{n} \sum_{j=0}^{n} c_{i} c_{j} e_{i} e_{j} \\
& \times\left(\left(k_{x i} k_{x j} s_{x i 0} s_{x j 0}+k_{y i} k_{y j} c_{x i 0} c_{x j 0}\right) c_{p a}^{2} s_{p a}^{2}\right. \\
& \left.+\frac{k_{x i}}{k_{y i}} k_{x j} k_{y j} s_{x i 0} s_{x j 0} s_{p a}^{4}+k_{y j}^{2} c_{x i 0} c_{x j 0} c_{p a}^{4}\right) .
\end{aligned}
$$

In this mode the horizontal and vertical planes are coupled:

$$
\begin{aligned}
\partial \theta_{x} / \partial y= & \partial \theta_{y} / \partial x=-\frac{8 L}{(B \rho)^{2} k^{2}} \sum_{i=0}^{n} \sum_{j=0}^{n} c_{i} c_{j} e_{i} e_{j} \\
& \times\left(-k_{x i} \frac{k_{x j}^{2}}{k_{y j}} s_{x i 0} c_{x j 0}+\frac{k_{x i}}{k_{y i}} k_{x j}^{2} s_{x i 0} c_{x j 0}\right. \\
& \left.+k_{y i} k_{x j} c_{x i 0} s_{x j 0}-k_{x j} k_{y j} c_{x i 0} s_{x j 0}\right) c_{p a}^{2} s_{p a}^{2} .
\end{aligned}
$$

These terms can be used for an on-line compensation of the tune shifts using storage ring quadrupoles. The coupling in the inclined mode can be minimized as well to keep the vertical emittance small (see Sec. V).

The rotation of the dynamic field integrals is given by $(\nabla \times \overrightarrow{\tilde{B}})_{z} \propto \partial \theta_{x} / \partial x+\partial \theta_{y} / \partial y$. This function is plotted for various phases in Fig. 5. For zero phase (nearly planar device) the rotation is constant over a large transverse region. The term cannot be compensated with a single quadrupole; however, this can be achieved with two quadrupoles with an appropriate phase advance in between. For a finite phase the rotation shows a significant fine structure in transverse direction and a compensation with quadrupoles is impossible. The fine structure is independent from the properties of 2D-correction elements such as shims (see the next section) since their contribution is rotation free.

\section{SHIMMING OF DYNAMIC FIELD INTEGRALS}

In Sec. III, we presented analytic descriptions of ideal periodic undulator fields. Real undulators employ field errors due to magnet block errors (dipole component and inhomogeneities) and geometric errors (fabrication and assembling errors). These effects which can be described by a $2 \mathrm{D}$ distribution of static field integrals have to be minimized, i.e., shimmed. The evaluation of a shim distribution for a given distribution of field integral errors is done via the solution of a set of linear equations which is a fast algorithm. For this purpose the response function of various shims at different transverse positions has to be
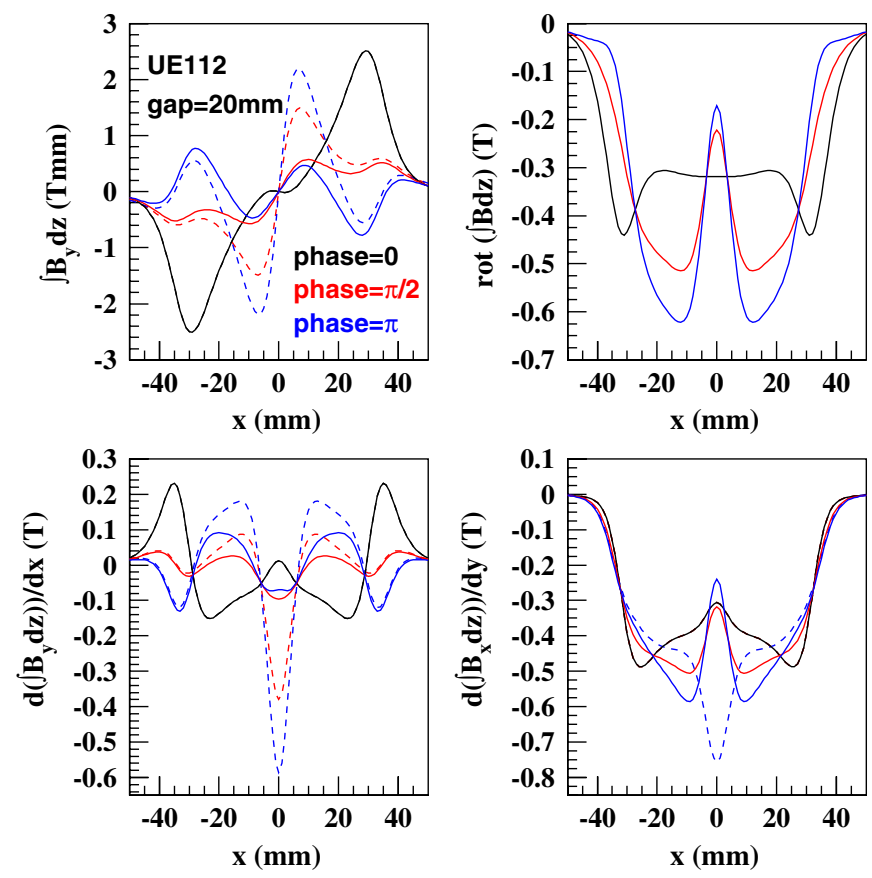

FIG. 5. Top left: Sum of dynamic and static field integrals of the unshimmed device (dashed) and the shimmed device (solid) for various phases. The $\mathrm{Fe}$ shims have a negligible effect at phase $=0$. Top right: Rotation of the dynamic field integrals (rotation of the shim contribution is zero) for the phases of the figure top left. Bottom left and right: Contributions to the rotation of the sum of dynamic and static field integrals of the unshimmed device (dashed) and the shimmed device (solid). All data are given for the UE112 at a gap of $20 \mathrm{~mm}$ in the midplane.

evaluated on the same grid as the error distribution. The shim response function can be obtained either by numerical techniques (e.g. with RADIA [35,36]) or by measurements. Principally, there is no limitation regarding the shimming accuracy since the shim response is represented by static $2 \mathrm{D}$-field integrals as well. The shimming technique is well established and we do not want to go into detail. However, we want to discuss the shim representation as used for tracking.

An expansion of insertion device field integral errors with respect to $2 \mathrm{D}$ multipoles in analogy to storage ring magnets is not appropriate since the expansion converges only on a source free disk. The radius of this disk is defined by the distance of the closest error to the undulator axis, e.g., half the magnetic gap $g$. In storage rings the horizontal travel of electrons reaches much further out than $g / 2$ and the multipole expansion diverges in this regions. We will present a field integral description which is not limited in the horizontal direction. This description is used in the particle tracking of Sec. II.

The dynamic field integrals can be compensated only by part with shims as discussed in Sec. IV. Nevertheless, shims reduce the dynamic effects significantly. The dynamic field integrals are shimmed in the midplane and the 
shim strength and shape are optimized for this case. In principle, a perfect compensation in the midplane is possible. This includes the horizontal and vertical dynamic field integrals as well as all derivatives with respect to the horizontal coordinate. The derivatives in the vertical direction are nonzero. However, usually the vertical emittance and betatron function are small and the residual errors are tolerable.

Shim field integrals for the compensation of static and dynamic field integrals have to be included in a particle tracking scheme to achieve realistic results. In the following we will derive analytic expressions for various types of shims which are suited for the tracking scheme of Sec. II.

\section{A. Analytic shim model}

Undulator fields have to meet two independent requirements: (i) The local on-axis field distribution has to be optimized with respect to the phase error [38] to provide highest photon brightness even at higher harmonics. (ii) The off-axis field integrals have to be minimized for a negligible impact of the undulator on the electron beam dynamics. This requirement is more stringent for storage ring devices and less critical for single pass devices.

Local field errors as well as field integral errors can be compensated, with pieces of iron [39-42], with permanent magnets [43], via a transverse movement of magnets, the so-called virtual shimming [44], or with special coils close to the electron beam [45]. Generally, the effect of a shim is the introduction of local transverse kicks (in horizontal and vertical direction) where the kick amplitudes depend on the transverse shim position and shim shape. A thorough magnet block characterization and sorting (based on dipole errors and inhomogeneities) and an accurate assembly provides already small phase errors [43]. The shimming effort concentrates on the 2D distribution of the integrated kicks.

Within a certain gap range $g=g_{0} \pm \Delta g$ the field integrals of any individual shim or shim distribution is approximately written as [18]

$$
\begin{aligned}
\bar{B}_{x}= & \sum_{i=0}^{n} \frac{k_{x i}}{k_{y i}}\left[\bar{c}_{i} \sin \left(k_{x i} x\right) \cosh \left(k_{y i} y\right)+\bar{s}_{i} \cos \left(k_{x i} x\right)\right. \\
& \left.\times \sinh \left(k_{y i} y\right)\right] \exp \left[-k_{y i} \Delta g / 2\right] \\
\bar{B}_{y}= & \sum_{i=0}^{n}\left[-\bar{c}_{i} \cdot \cos \left(k_{x i} x\right) \sinh \left(k_{y i} y\right)+\bar{s}_{i} \sin \left(k_{x i} x\right)\right. \\
& \left.\times \cosh \left(k_{y i} y\right)\right] \exp \left[-k_{y i} \Delta g / 2\right] \\
k_{x i}= & k_{y i} .
\end{aligned}
$$

At a given gap $g=g_{0}$, i.e. $\Delta g=0$, the expansion converges since the expansion functions represent a complete set of functions for all 2D distributions of field integrals which obey the 2D-Maxwell equations. For other gaps $(\Delta g \neq 0)$ the accuracy depends on the specificity of the shim (see below).
The number of terms is reduced if a specific shim symmetry is assumed. In the elliptical mode of an APPLE II undulator, the required shim configuration for the minimization of the dynamic field integrals follows Eq. (71) with $\bar{c}_{i}=0, \forall i$ (point symmetry with respect to the undulator axis). For this specific case the shims are grouped in quadruples. One quadruple consists of four shims, one shim on each of the four magnet rows. Each quadruple is described by Eq. (71).

Usually, the coefficients $\bar{s}_{i}$ and $\bar{c}_{i}$ are evaluated at the smallest operation gap $g_{0}$ because then, the simulation accuracy is highest in the gap regime where the dynamic field integrals and the response of the shims are largest. The shim field integrals are evaluated at $y=0$ within a transverse range of $\lambda_{x 1}=2 \pi / k_{x 1}$ and the Fourier components are derived from this distribution where $k_{x i}=k_{y i}=i \cdot k_{x 1}$.

Equation (71) is well suited for situations where a linear superposition of fields is justified such as permanent magnet shims on top of the periodic structure [37], magic fingers at the undulator ends [43], virtual shims [44], or flat wires as realized for the BESSY II UE112. Fe shims have a small coercivity and, thus, have a response function which depends strongly on the magnetic environment, i.e., the shim response is magnet row phase dependent. In fact, this behavior is used to compensate for phase dependent static field integrals of APPLE II undulators which are due to a finite magnetic susceptibility [43]. Furthermore, due to the cross talk between the upper and lower magnet girders the gap dependence of $\mathrm{Fe}$ shims deviates from Eq. (71).

We extend the ansatz of Eq. (71) in order to describe Fe shims within the complete phase and gap range of an APPLE II undulator. Without losing generality we regard only the field integral differences with respect to the $\varphi_{1}=$ $\varphi_{2}=0$ geometry, where the field integrals are usually compensated with permanent magnet shims (see Sec. V B). At a given gap the phase dependent transverse distribution of the dynamic field integrals in the elliptical (inclined) mode can be described by two (four) generic functions [Eqs. (65) and (66)]. The vertical field integrals increase from zero to a maximum going from $\varphi_{1}=0$ to $\varphi_{1}=\pi$. In the inclined mode the horizontal field integrals are zero for $\varphi_{2}=0$ and $\varphi_{2}=\pi$ and have a maximum in between. We are seeking a Fe-shim configuration with approximately the same phase dependence. Chavanne et al. proposed $L$-shaped $\mathrm{Fe}$ shims to compensate the dynamic field integrals in the elliptical mode [46]. In the inclined mode the $L$ shims enhance the dynamic field integrals, however. Following Chavanne's idea, four identical shims with a length of half a period are placed at the magnet edges close to the undulator axis (standard $L$-shim quadruple). The shims are centered on the vertically magnetized blocks. Looking downstream the shims are placed on negative blocks (producing a negative vertical field on 

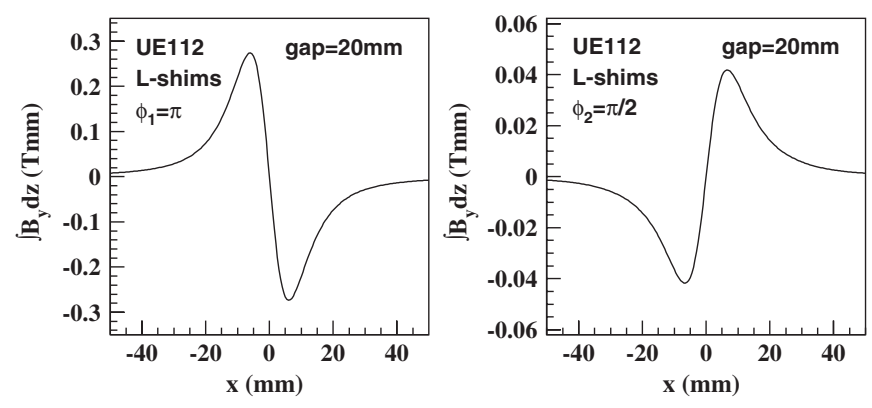

FIG. 6. Generic functions for the description of the $L$ shims. Plotted is the amplitude of a standard $L$-shim quadruple for the UE112 (see text).

axis) at the right-hand side and on positive blocks at the left-hand side (an electron machine is assumed). The static field integrals of this shim configuration have the required point symmetry. Numerical simulation shows that an $L$-shim quadruple can be represented by two generic functions which represent the vertical field integral distribution at $\varphi_{1}=\pi$ (elliptical mode) and the horizontal field integral distribution at $\varphi_{2}=\pi / 2$ (inclined mode). A Fourier decomposition of the two functions yields two sets of Fourier coefficients $\bar{c}_{i}$ and $\bar{s}_{i}$, where the $\bar{c}_{i}$ terms are zero in this geometry. An example of the generic functions for the UE112 is given in Fig. 6.

The generic $L$-shim functions have to be scaled appropriately with phase and gap dependent functions $R$ and $S$, where $R$ and $S$ are related to the regular and skew multipole components. We chose the following ansatz for the $L$-shim quadruple description:

$$
\begin{aligned}
\bar{B}_{x}= & \sum_{i=0}^{n}\left[S \bar{c}_{i} \sin \left(k_{x i} x\right) \cosh \left(k_{x i} y\right)+R \bar{s}_{i} \cos \left(k_{x i} x\right)\right. \\
& \left.\times \sinh \left(k_{x i} y\right)\right] \exp \left[-k_{x i} \Delta g / 2\right] \\
\bar{B}_{y}= & \sum_{i=0}^{n}\left[-S \bar{c}_{i} \cos \left(k_{x i} x\right) \sinh \left(k_{x i} y\right)+R \bar{s}_{i} \sin \left(k_{x i} x\right)\right. \\
& \left.\times \cosh \left(k_{x i} y\right)\right] \exp \left[-k_{x i} \Delta g / 2\right] \\
R(\varphi, \Delta g)= & a_{1}(\varphi)\left\{b_{1}(\varphi)+\left[1-b_{1}(\varphi)\right] \exp \left[-c_{1}(\varphi) \Delta g\right]\right\} \\
S(\varphi, \Delta g)= & a_{2}(\varphi) \exp \left[-c_{2}(\varphi) \Delta g\right] .
\end{aligned}
$$

Equation (72) includes different Fourier coefficients and different functions $R$ and $S$ in the elliptical and inclined mode. $a_{1}(\varphi)$ and $a_{2}(\varphi)$ describe the phase dependency at smallest gap $g_{0}$ and they have to be determined from numeric field simulations at this gap. For parametrization they can be decomposed into Fourier components with respect to the phase parameter. Numerical simulations show that the functions $a_{1}(\varphi)$ are similar in the elliptical and inclined mode. $a_{2}(\varphi)$ is nonzero only in the inclined mode. At larger gaps the field integrals of Eq. (72) scale with an exponential expression containing the factors $k_{x i}$. Residual errors are visible in Fig. 7. They can be described by the phase dependent function $b_{1}$ and the gap dependent
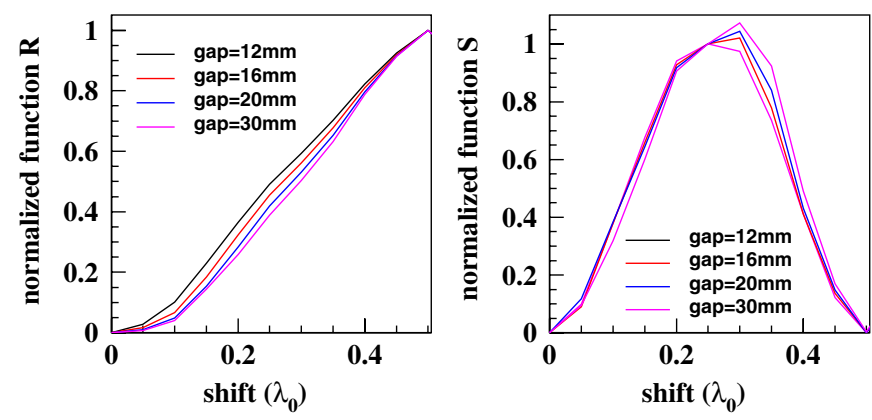

FIG. 7. Gap and shift dependence of the functions $R$ (left) and $S$ (right). $R$ is normalized to one at shift $=0.5 \lambda_{0} . S$ is normalized to one at shift $=0.25 \lambda_{0}$. The data are given for the elliptical mode.

functions $c_{1 / 2}$. The functions $a_{1,2}, b_{1}$, and $c_{1,2}$ are plotted in Fig. 8 for the BESSY II UE112 undulator. With a gap of $g_{0}=20 \mathrm{~mm}$ and $\lambda_{x 0}=400 \mathrm{~mm}, 80$ Fourier coefficients have been derived for the UE112. Using these coefficients the field integrals in the midplane are evaluated with an accuracy well below $1 \%$ for vertical field integrals and $2 \%$ for the horizontal field integrals in the inclined mode over a gap range of 20-100 mm (Fig. 9). These errors are related to the maximum field integral value for all phases at $g_{0}=$ $20 \mathrm{~mm}$. The errors decrease for increasing gaps.

In tracking simulations this model should be checked for the most critical situations (e.g. smallest gap and $\varphi_{1}=\pi$ ) versus a simulation based on Fourier coefficients specifically derived for this gap and phase. Figure 10 shows errors of only $10 \%$ even $8 \mathrm{~mm}$ above midplane for specifically evaluated coefficients (gap $=20 \mathrm{~mm}$, inclined mode, $\varphi_{2}=\pi / 2$ ). With smaller vertical offsets the errors decrease.

In other cases, e.g., during evaluation of the correction currents for active compensation of the dynamic field integrals (see Sec. VC), only the field integrals in the midplane are required. Then, it is adequate for accuracy and advantageous concerning CPU time (no numeric field simulation required) to extrapolate the field integrals at various gaps $g=g_{0}+\Delta g$ from the coefficients derived for smallest gap $g_{0}$ using Eq. (72).
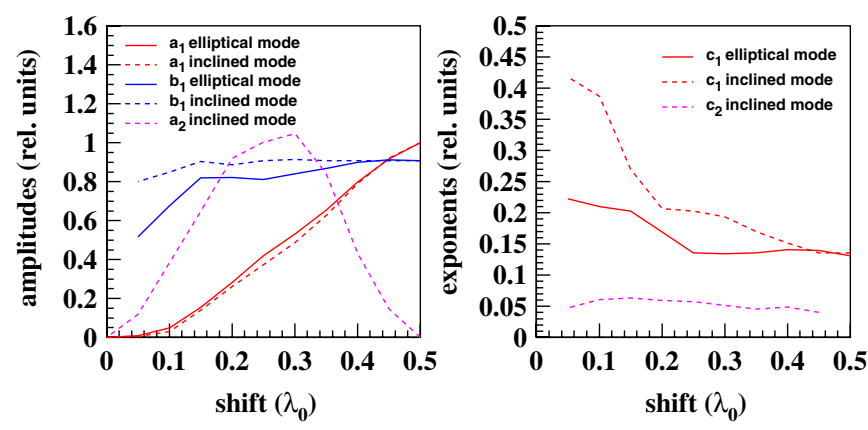

FIG. 8. Parameters of the $L$-shim model depending on the row phase. The parameters are given for the UE112 at a reference gap of $20 \mathrm{~mm}$. 

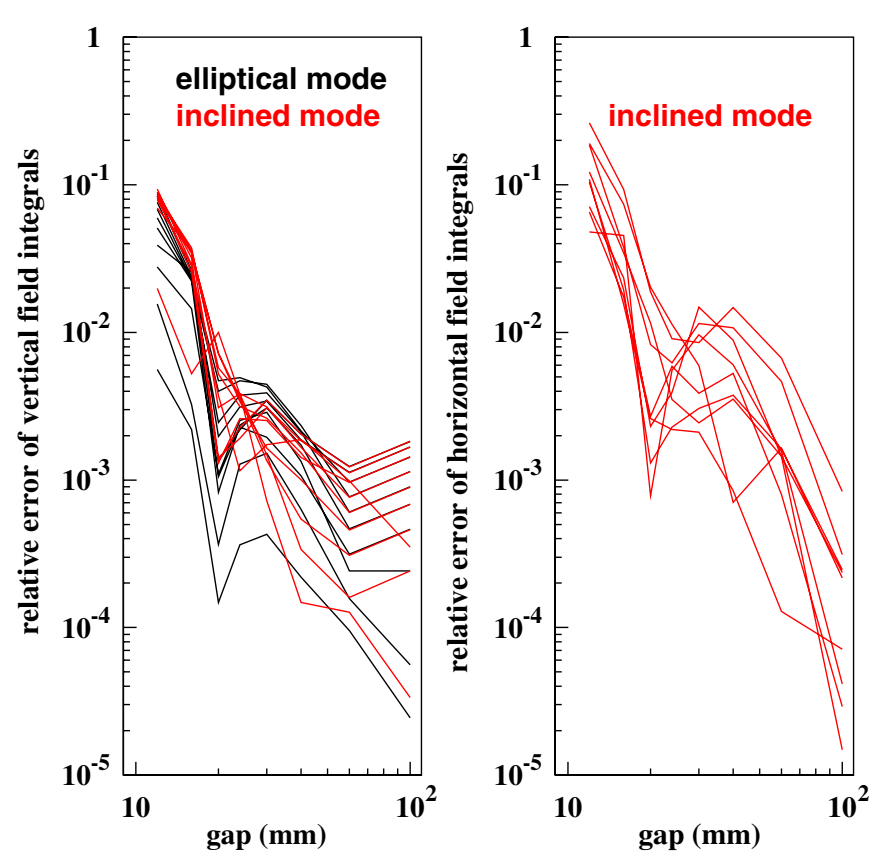

FIG. 9. Relative error of the field integral prediction for various gaps and shifts and for the elliptical and the inclined mode as evaluated from the shim model (see text). The absolute error is defined by the difference between the field integrals from the model and field integrals from RADIA. The relative errors are defined by the ratio between the absolute errors and the maximum field integral value at $20 \mathrm{~mm}$ gap and shift $=0.5 \lambda_{0}$ for the vertical field integrals and shift $=0.25 \lambda_{0}$ for the horizontal field integrals.

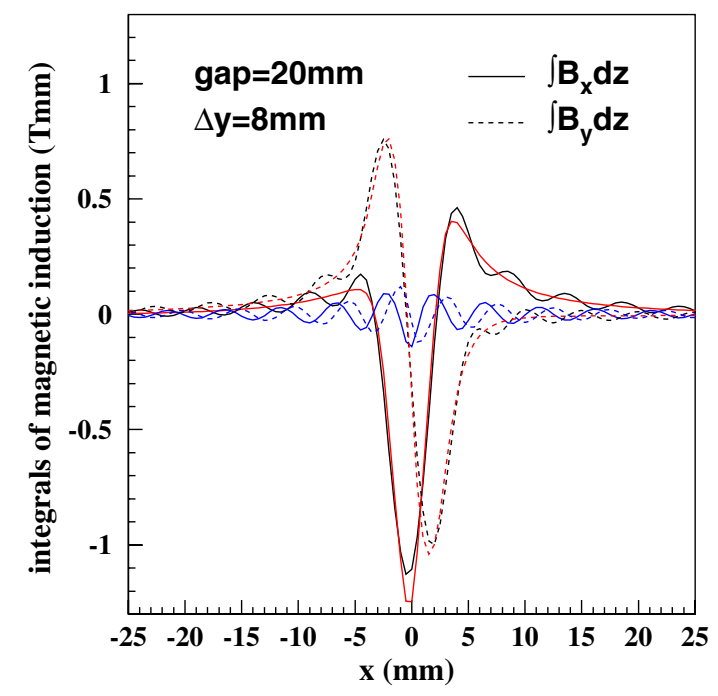

FIG. 10. Off plane accuracy of the $L$-shim field integrals in the inclined mode. The data are evaluated for a standard $L$-shim quadruple (see text) of the UE112 $8 \mathrm{~mm}$ above the midplane with gap $=20 \mathrm{~mm}$, shift $=0.25 \lambda_{0}$. Black: Exact values as evaluated with RADIA. Red: Analytic model with 80 Fourier coefficients. The expansion ranges goes from $-200 \mathrm{~mm}$ to $+200 \mathrm{~mm}$ with a spacing of $0.5 \mathrm{~mm}$. Blue: Residuals.

\section{B. Passive shimming in the elliptical mode}

The passive shimming of APPLE II undulators is done in four steps [43]: (i) virtual shimming for trajectory straightening; (ii) shimming of phase dependent field integrals with flat $\mathrm{Fe}$ shims of $4 \mathrm{~mm}$ width which are distributed in transverse direction on a grid with a grid size of $4 \mathrm{~mm}$; (iii) shimming of static field integrals using so-called magic fingers at either end of the undulator. The magic fingers are arrays of permanent magnets with a cross section of $4 \times 4 \mathrm{~mm}^{2}$ and variable length distributed on a transverse grid with grid size of $4 \mathrm{~mm}$; (iv) passive shimming of the dynamic field integrals with $L$ shims. The $L$ shims are glued onto the structures in the storage ring tunnel. This procedure has successfully been applied to all BESSY II APPLE II undulators [37]. The geometrical shape of the $L$ shims has been optimized such that the transverse distribution of the dynamic field integrals is minimized at the smallest gap of the individual undulator. The horizontal and vertical sizes of the BESSY-II $L$ shims are 2 and $4 \mathrm{~mm}$, respectively. The influence of the shape is discussed in detail for the BESSY II UE112 in [18]. Shims with varying thicknesses and lengths for the elliptical mode are discussed in that paper but it has been concluded that the additional effort with different shim types does not pay since the $L$ shims do not help in the inclined mode. The strong dynamic field integrals in the inclined mode have to be compensated actively (Sec. VC), and once an active compensation scheme is available also the residual errors in the elliptical mode can be minimized. Nevertheless, the passive shims are extremely useful in the elliptical mode since the main part of the dynamic multipoles is canceled even if the active compensation fails due to a hardware error. The spectral power of the optimized $L$-shim configuration in the midplane at smallest gap and maximum phase is plotted in Fig. 11.

The $L$ shims are optimized for smallest gap and $\varphi_{1}=\pi$. In the following we estimate the quality of the $L$-shim compensation at other gaps $g=g_{0}+\Delta g$. We assume equal expansion ranges of the Fourier decomposition for the shims and the single magnet row (needed for evaluation of dynamic field integrals). The considerations are based on the assumption of $R \approx 1$ which is accurate within 5\% at maximum shift and a gap range between 20 and $40 \mathrm{~mm}$. For small period lengths the approximation is not valid, but in that case the dynamic field integrals are small and, usually, are not shimmed.

In the vertical linear mode with $\varphi_{1}=\pi$ the static kicks $\bar{\theta}_{x}$ in the midplane which are due to the shim field integrals have a gap scaling of [Eq. (71)]

$$
\bar{\theta}_{x} \propto \sum_{m=1}^{n} \bar{A}_{m} e^{-m k_{x 1} \Delta g / 2} \sin \left(m k_{x 1} x\right) .
$$

On the other hand, the kicks of the dynamic field integrals $\tilde{\theta}_{x}$ scale with [Eq. (65)] 


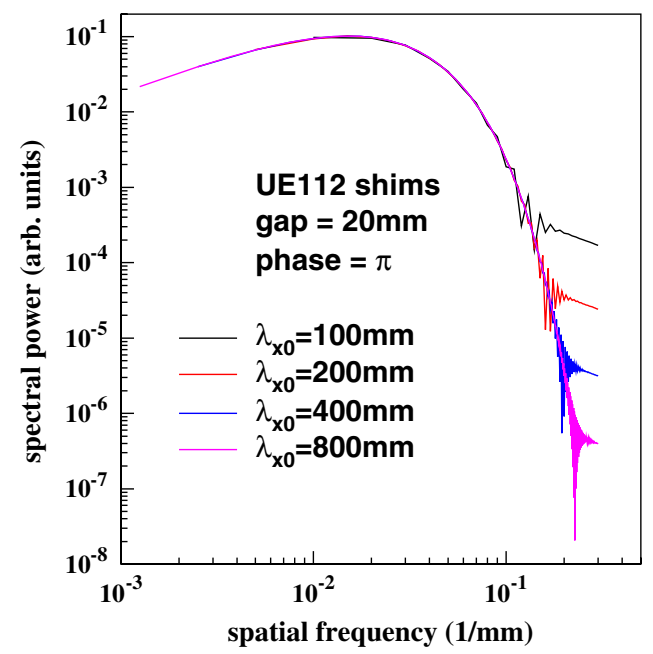

FIG. 11. Spectral power of the transverse field integral distribution of a standard UE112 L-shim quadrupole for expansion ranges between 100 and $800 \mathrm{~mm}$ (number of Fourier coefficients between 30 and 240). The plot shows the robustness of the expansion procedure with respect to the range of expansion. The high frequency cutoff is defined by the ratio of the number of coefficients and the expansion range. The accuracy at high frequencies improves with a bigger expansion range.

$$
\begin{aligned}
\tilde{\theta}_{x}= & \sum_{i=0}^{n} \sum_{j=1}^{n} \cdot \tilde{A}_{i j}\left(\varphi_{1}\right) \cdot e^{-\left[\left(k_{y i}+k_{y j}\right) \Delta g / 2\right]} \\
& \times \cos \left(i \cdot k_{x 1} x\right) \sin \left(j \cdot k_{x 1} \cdot x\right) \\
= & \sum_{i=0}^{n} \sum_{\substack{j=1 \\
j \geq i}}^{n} \cdot 2^{-l} \cdot \tilde{A}_{i j}\left(\varphi_{1}\right) \cdot e^{-\left[\left(k_{y i}+k_{y j}\right) \Delta g / 2\right]} \\
& \times \sin \left[(i+j) \cdot k_{x 1} \cdot x\right] \\
l= & 1, \quad \forall j=i \quad l=2, \quad \forall j>i \\
\tilde{\theta}_{x}= & \sum_{\substack{m=1 \\
m=1}}^{2 n} \tilde{A}_{m}\left(\varphi_{1}\right) \sin \left(m \cdot k_{x 1} \cdot x\right) \\
\tilde{A}_{m}\left(\varphi_{1}\right)= & \sum_{\substack{i=0, j=1 \\
j=i \\
i+j=m}}^{n} 2^{-l} \cdot A_{i j}\left(\varphi_{1}\right) \cdot e^{-\left[\left(k_{y i}+k_{y j}\right) \Delta g / 2\right]}
\end{aligned}
$$

The shim shape is optimized such that the dynamic field integrals at smallest gap are compensated in the midplane:

$$
\sum_{m=1}^{n} \bar{A}_{m} \sin \left(m \cdot k_{x 1} \cdot x\right) \approx \sum_{m=1}^{2 n} \tilde{A}_{m}\left(\varphi_{1}\right) \sin \left(m \cdot k_{x 1} \cdot x\right),
$$

which requires for each individual term $\bar{A}_{m} \approx \tilde{A}_{m}\left(\varphi_{1}\right)$. The gap dependence of the kicks is included in the exponential terms:

$$
\bar{A}_{m} \cdot e^{-m \cdot k_{x 1} \cdot \Delta g / 2} \approx \tilde{A}_{m}\left(\varphi_{1}\right) \cdot e^{-\left(m \cdot k_{y 1}\right) \Delta g / 2} .
$$

For small $k$ which means large period lengths we expand:

$$
m \cdot k_{y 1}=\sqrt{m^{2} \cdot k_{x 1}^{2}+k^{2}} \approx m \cdot k_{x 1} \cdot\left(1+\frac{k^{2}}{2 \cdot m^{2} \cdot k_{x 1}^{2}}\right) .
$$

As an example we examine the BESSY UE112 with $\lambda_{0}=112 \mathrm{~mm}$. With a region for the transverse Fourier expansion of $\pm 100 \mathrm{~mm}$ we can set $m \cdot k_{y 1} \approx m \cdot k_{x 1}$ with an error below $18 \%$ (10\%) for the Fourier terms $m \geq 3$ $(m \geq 4)$. According to Eq. (76) we have the same gap dependence of the Fourier components $m \geq 3$ of the dynamic field integrals and the static field integrals of the shims. Restricting the discussion to the disk of convergence the multipole content of the terms in Eq. (75) is derived from Eq. (78):

$$
\sin \left(m \cdot k_{x 1} \cdot x\right)=\sum_{i=1}^{\infty} \frac{(-1)^{i} m^{\tilde{i}}}{\tilde{i} !}\left(k_{x 1} x\right)^{\tilde{i}} \quad \tilde{i}=2 \cdot i-1,
$$

where the terms proportional $x, x^{3}, x^{5}, x^{7}$ are attributed to a quadrupole, octupole, dodecapole, and hexadecapole. Numerical simulations show that only the coefficients $m \geq 3$ contribute significantly to the multipoles higher than the quadrupole (Fig. 12). This is the reason for a similar gap dependence of the static and dynamic multipoles for long period devices. The quadrupole terms scale differently, but they can easily be compensated with a tune feed forward scheme.

Figure 13 compares the UE112 with a UE56 with the same length. The $L$ shims of both devices have roughly the same gap dependence. Differences are due to the different cross talk between upper and lower girder. The strength of the dynamic field integrals scales quadratically with the period length. The dynamic field integrals of the UE56 drop much faster than those of the UE112. As expected the $L$ shims compensate the dynamic multipoles of the UE112 over a

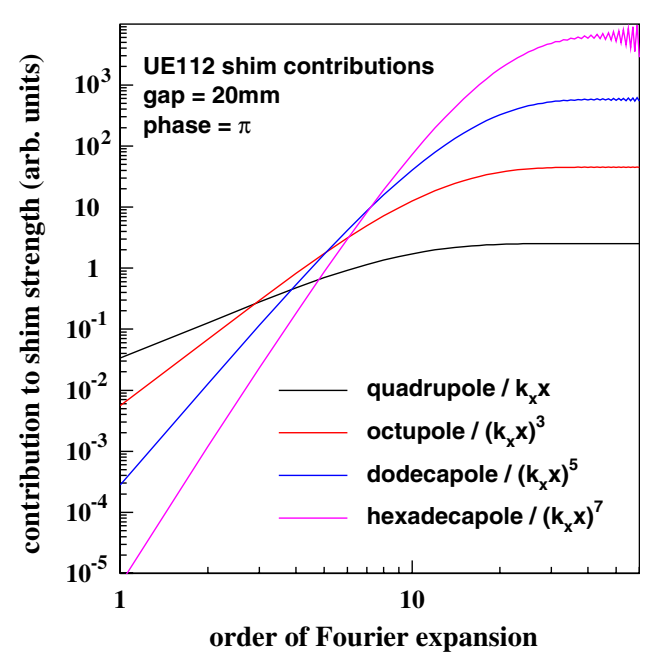

FIG. 12. Contributions from different Fourier components to the lowest order multipoles of the $L$ shims. With increasing multipole order the contributions of low order Fourier components decrease. This is the reason for the similar gap scaling of $L$ shims and dynamic multipoles (see text). 

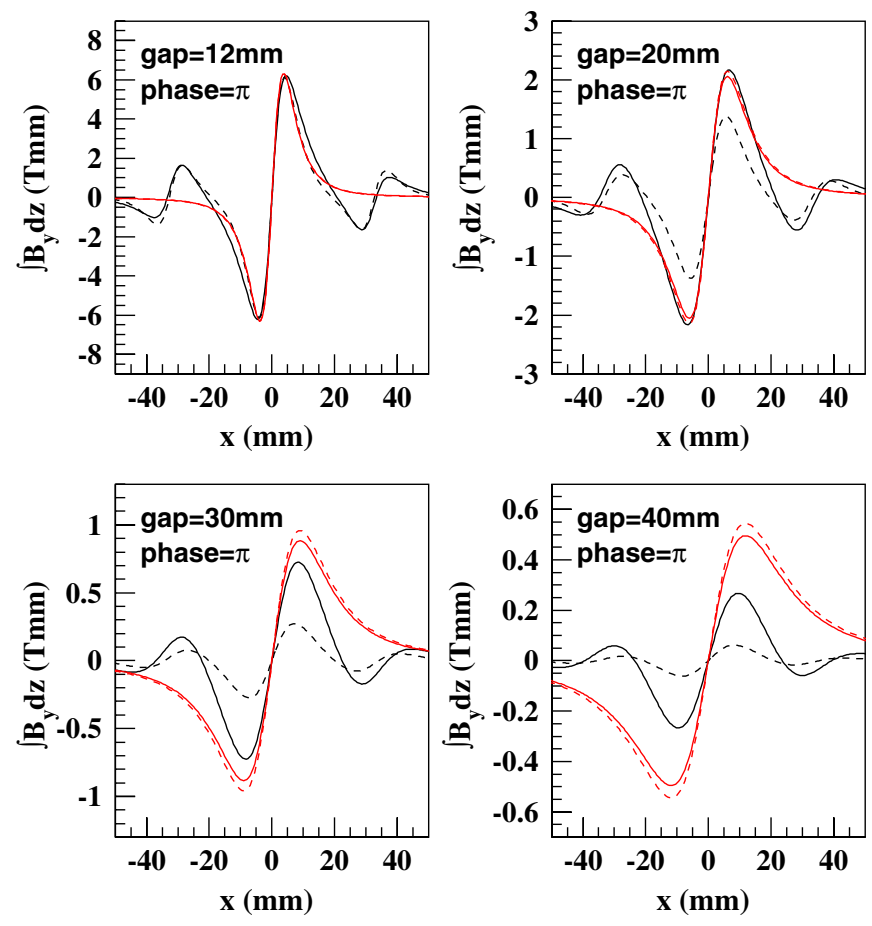

FIG. 13. Dynamic multipoles (black) and $L$ shims (red) for the UE112 (solid) and a UE56 with the same length (dashed) in the midplane for different gaps. Dynamic multipoles and shims of the UE56 are scaled with a constant factor for better comparison. The shim configuration (number of standard $L$-shim quadruples and shim shape) has been optimized for the UE112 at a gap of $20 \mathrm{~mm}$.

considerable gap range which is not the case for the UE56. At large gaps the relative accuracy gets worse even for the UE112; however, the absolute values are small. As expected, the compensation of the dynamic multipoles of short period devices is less accurate; however, the terms are smaller.

As an example for the generating function-based tracking routine of Sec. II and the analytic models of the undulator and the $L$ shims of Secs. III and V we present results from the UE112. The dynamic aperture of the UE112 undulator was explored at smallest gap of $20 \mathrm{~mm}$ and at maximum shift without and with $L$ shims [17]. The dynamic kicks to a particle when passing the undulator with different initial conditions are plotted in Fig. 14. The initial horizontal phase space variables $x$ and $x^{\prime}$ of the particles were equally distributed on a phase space ellipse with semiaxes of $30 \mathrm{~mm}$ and $2 \mathrm{mrad}$. The kicks can be reduced significantly with the implementation of $L$ shims (Fig. 14). Only a linear dependence on the transverse displacement with a minor modulation is left which can be compensated with an appropriate quadrupole tuning. The horizontal phase space without $L$ shims is reduced by about a factor of 2 as compared to the case with undulator gap open. The horizontal dynamic aperture is below $15 \mathrm{~mm}$ and a spurious dilution of the phase space is observed at even smaller displacements (Fig. 15). The horizontal phase space can be recovered with $L$ shims as shown in Fig. 15.

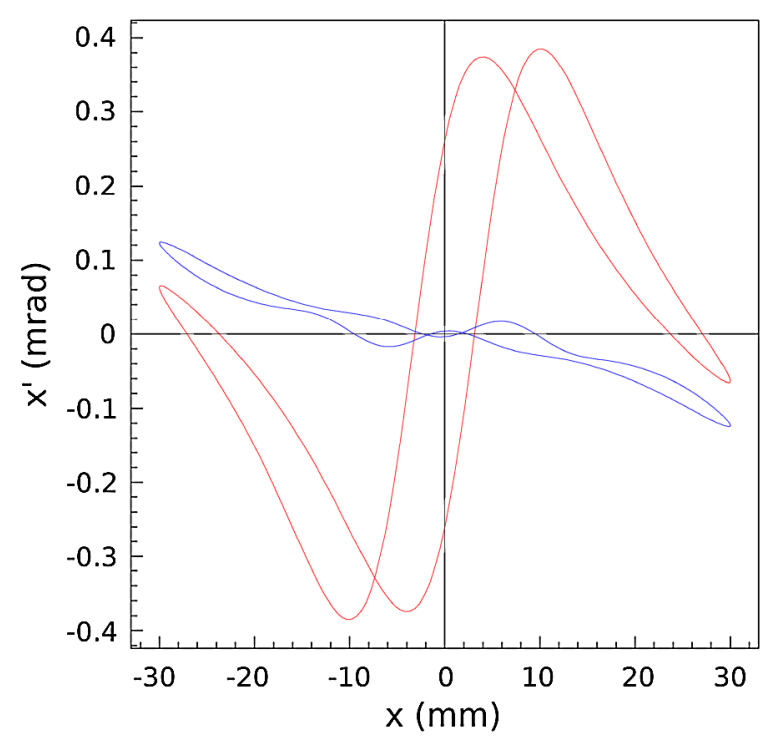

FIG. 14. Horizontal kick in mrad and displacement in $\mathrm{mm}$ for an electron passing the UE112 at $20 \mathrm{~mm}$ gap and maximum phase for initial variables $x$ and $x^{\prime}$ equally distributed on a phase space ellipse (see text). The red line corresponds to the unshimmed device and the blue line shows the results with an $L$-shim compensation.

\section{Active shimming in the inclined mode}

As shown in Sec. IV the dynamic field integrals scale with the square of the period length and inversely with the square of the beam energy. The UE112 operated at BESSY II (beam energy of $1.7 \mathrm{GeV}$ ) produces dynamic field integrals up to $3 \mathrm{Tmm}$. They can be compensated with $L$ shims in the elliptical mode. In the inclined mode no passive compensation scheme is available and an active scheme has to be adopted. The highest flexibility is provided by a set of wires with an individual excitation. At BESSY two sets of 14 wires each are glued to the vacuum chamber above and below midplane. The transverse width of the wires is $3 \mathrm{~mm}$ allowing for a relative distance of $4 \mathrm{~mm}$. The wires are grouped in pairs and operated by 14 power supplies. In this geometry point symmetric field integral distributions of nearly arbitrary shape can be produced $[18,45]$. The complex field integrals $\hat{\bar{B}}$ of a wire depending on the complex coordinates $\hat{r}_{(0)}$ are given by

$$
\begin{aligned}
\hat{\bar{B}}(\vec{r}) & =i d \frac{\hat{r}_{0}-\hat{r}}{\left|\hat{r}_{0}-\hat{r}\right|^{2}} & \hat{\bar{B}} & =\bar{B}_{x}-i \bar{B}_{y} \\
\hat{r}_{(0)} & =x_{(0)}-i y_{(0)} & d & =\frac{\mu_{0}}{2 \pi} I L .
\end{aligned}
$$

$I$ is the current and $L$ is the length of the wire. The field integrals can be decomposed into regular and skew multipoles [Eqs. (80) and (81)] [45]. The multipole expansion of Eqs. (80) and (81) converges only on a source-free circular disk. At distances larger than the distance to the wire it diverges [45]. Outside the disk of convergence the wires have to be described by Eq. (71): 

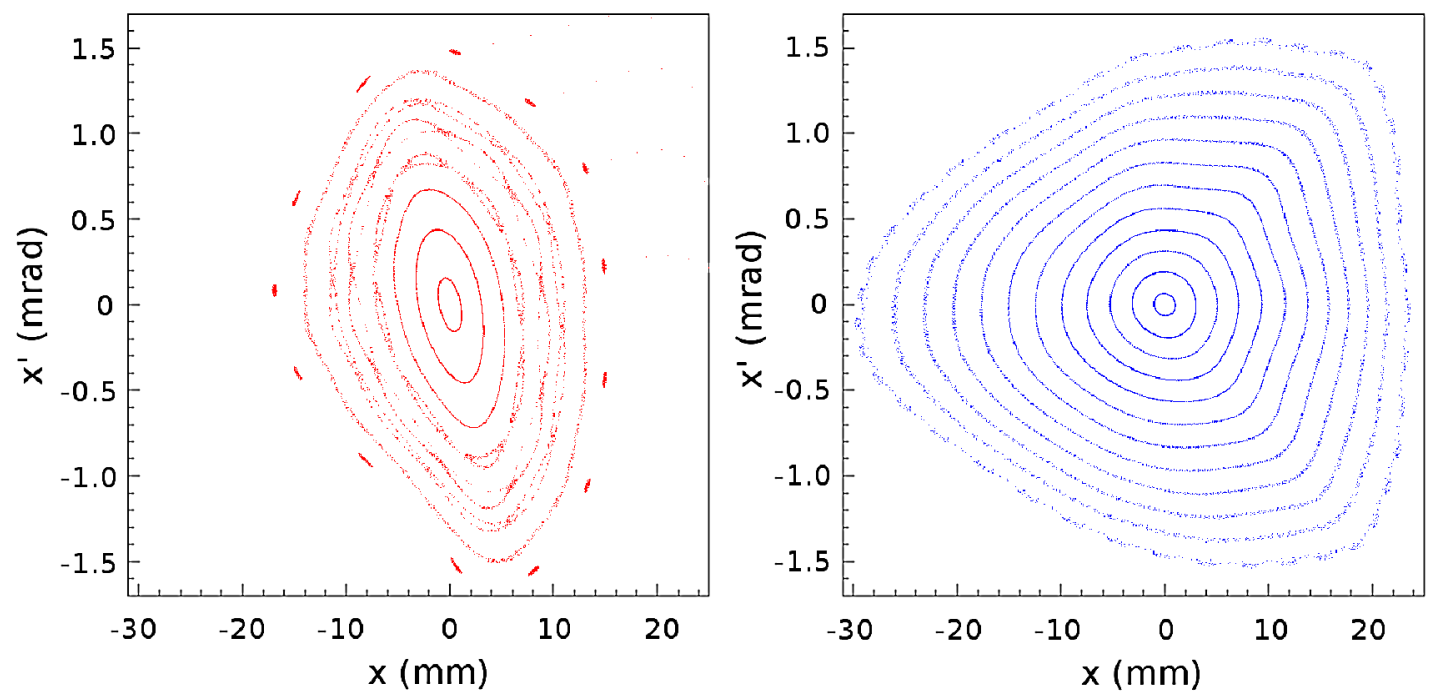

FIG. 15. Horizontal phase space plot of 1000 turns and increasing horizontal starting amplitude at nominal momentum. The vertical starting amplitude is always $1 \mathrm{~mm}$. The dynamic field integrals are not compensated, left, and compensated by $L$ shims, right.

$$
\begin{aligned}
\bar{B}_{y} & =-\sum_{m=0}^{\infty} x^{m} \cdot d\left(\sum_{i=i \text { start }}^{m+1} \frac{c_{i j}}{r_{0}^{2 i}} \cdot x_{0}^{2[[m / 2]+i-m-1]-\bmod (m, 2)+1}-h(m) \sum_{i=i s t a r t-\bmod (m, 2)}^{m} \frac{c_{i j_{1}}}{r_{0}^{2 i}} \cdot x_{0}^{2[[(m-1) / 2]+i-m]-\bmod [(m-1), 2]}\right) \\
\bar{B}_{x} & =\sum_{m=0}^{\infty} x^{m} \cdot d \cdot y_{0} \cdot \sum_{i=i \text { istart }}^{m+1} c_{i j} \cdot \frac{1}{r_{0}^{2 i}} x_{0}^{2([m / 2\rceil+i-m-1)-\bmod (m, 2)}, \\
i \text { start } & =\lceil m / 2\rceil+1 \quad r_{0}^{2}=x_{0}^{2}+y_{0}^{2} \quad j=\bmod (m, 2)+2(i-\text { istart })+1 \quad j_{1}=\bmod (m, 2)+2(i-\text { istart })+2 \\
c_{i j_{(1)}} & =\left(\begin{array}{c}
i-1 \\
j_{(1)}-1
\end{array}\right) \cdot(-1)^{i+j_{(1)}-2} \cdot 2^{j_{(1)}-1} \quad h(m)=1-\delta_{m, 0} .
\end{aligned}
$$

In Sec. IV the dynamic field integrals are derived quantitatively. Based on these values the excitation patterns for the correction wires have been evaluated. The successful operation of the wires has been demonstrated for the UE112 [45]: In the elliptical and inclined mode the tune variations in the midplane are compensated, the coupling between planes in the inclined mode is minimized, and the injection efficiency in the inclined mode is recovered.

Below $100 \mathrm{eV}$ beam lines operated at APPLE II undulators profit from the universal mode. In this energy regime the optical elements in a beam line significantly change the polarization state of the incoming light. These modifications may change over time if the mirrors or gratings get contaminated. In the universal mode the undulator is capable to produce any state of polarization thus compensating polarizing beam line effects. As pointed out in Sec. IV this operation mode introduces a complicated dynamic field integral distribution lacking any symmetry. Pairing the correction wires as realized for the UE112 does not provide enough flexibility for the compensation. An individual powering of each wire is required. In the case of the
BESSY II UE112 the maximum correction current in the universal mode at a gap of $20 \mathrm{~mm}$ amounts to $25 \mathrm{~A}$, whereas in the inclined mode it is limited to $12 \mathrm{~A}$ [28]. The wires have been tested up to $16 \mathrm{~A}$, and, maybe, the wire thickness has to be increased slightly for the universal mode (2011, the wire thickness is $0.3 \mathrm{~mm}$ ). Within the limitations already discussed in Sec. IV the dynamic field integrals can be compensated with the wires. In practice it is not feasible to set up feed forward tables for the correction currents which span the whole parameter space in the universal mode (three parameters: two phases and gap). A better way is the on-line evaluation of the dynamic field integrals and the on-line derivation of the correction currents. Based on the equations in Appendix E a fast evaluation of the dynamic kicks is possible and the determination of the corrections currents requires only a matrix inversion. The UE112 has been operated in the universal mode and the impact on the storage ring has been studied in detail by measuring the tune shifts for a transversally displaced electron beam [28]. Extracting the dynamic kicks from the tune shifts, the agreement between 
prediction from the analytic model and the measurements is found to be better than $20 \%$ (normalized to the maximum values). Uncertainties of the analytic model and the measurements are the total strength of the $L$ shims (geometric errors are within $10 \%$ ), the actual betatron function at the undulator location, and the calibration of the beam position monitors at large horizontal displacements of the electron beam. Further improvements of the model are expected from a fine-tuning of the shim strength based on a higher measurement accuracy. The test of the improved model on the basis of tune measurements with a displaced electron beam as well as the implementation and operation of 28 independent correction wires will be the subject of future studies.

\section{CONCLUSION}

A symplectic, analytic, and fast particle tracking scheme which is based on generating functions has been presented. The scheme makes use of an analytic magnetic field model. Accurate analytic field descriptions of an APPLE II undulator as well as of shims for field compensation have been developed. Different undulators require only related Fourier coefficients. Different undulator settings such as undulator length, peak fields, magnetic gap, and magnet row phase (APPLE II) parameters are handled by the tracking routine in a parametrized model. This flexibility together with the fast tracking algorithm makes a scan of undulator parameters very efficient. The high accuracy permits the prediction of many features of the undulator electron beam interaction from tracking simulations. Furthermore, analytic expressions of dynamic field integrals, tune shifts, and coupling terms have been derived. These equations are essential for a fast active compensation of tune and higher order dynamic multipoles in arbitrary operation modes of complex undulators. Passive and active shimming concepts have been presented and it is concluded that APPLE II undulators with a long period length of $112 \mathrm{~mm}$ can be operated in a low energy storage ring such as BESSY II.

\section{ACKNOWLEDGMENTS}

The authors would like to thank A. Gaupp and M. Scheer for many fruitful discussions.

\section{APPENDIX A: REDUCE ROUTINE TO EVALUATE GENERATING FUNCTIONS FOR ARBITRARY FIELDS}

This REDUCE code solves iteratively the series expanded Hamilton-Jacobi equation to order 'ord'. The procedure 'tay3(func, $\operatorname{var} 1$, $\operatorname{var} 2$, $\operatorname{var} 3, \mathrm{n}$ )' is a selfwritten Taylor series expansion (not part of the REDUCE package). It expands the function 'func' with respect to the variable 'var1', 'var2', 'var3' to the order 'n'.

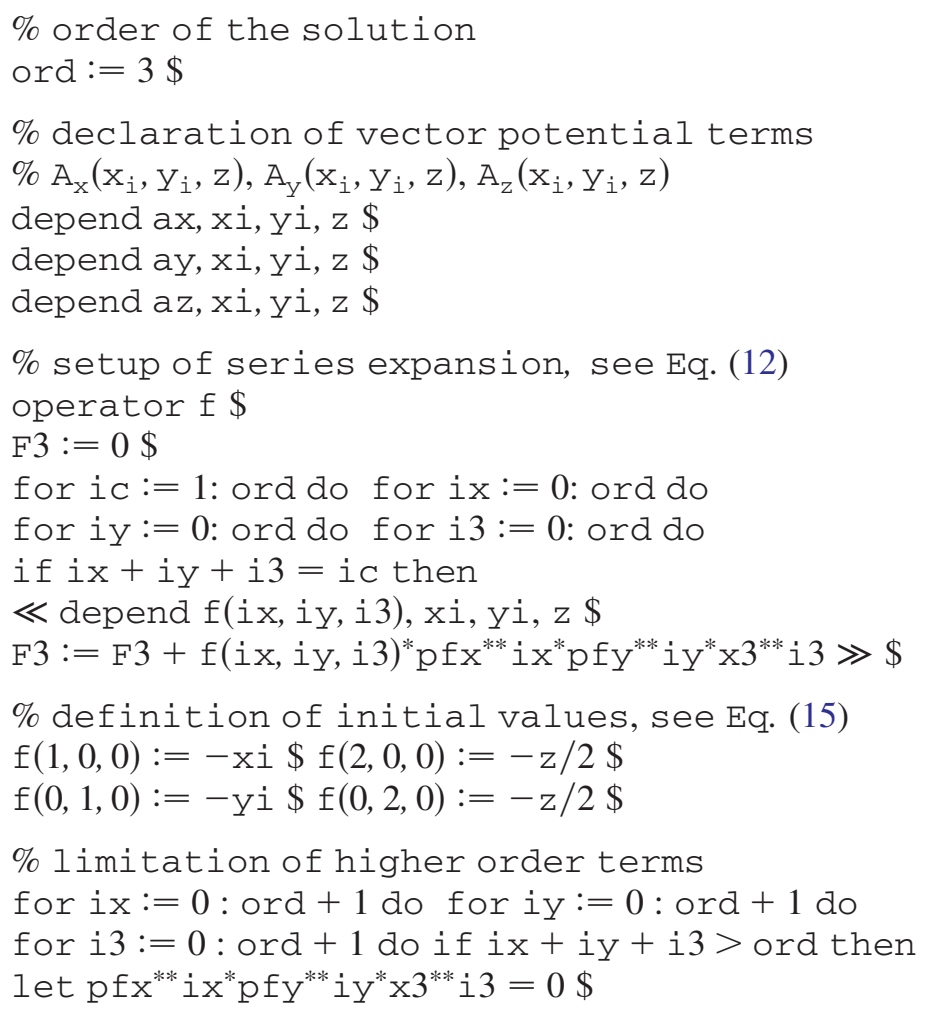


\% Taylor expansion of Hamiltonian to order 'ord'

hamc $:=-\left(\operatorname{sqrt}\left(-\left(\mathrm{pix}-\mathrm{x} 3^{*} \mathrm{ax}\right)^{* *} 2-\left(\mathrm{piy}-\mathrm{x} 3^{*} a y\right)^{* *} 2+1\right)+a z^{*} \mathrm{x} 3\right) \$$

ham := tay3(hamc, pix, piy, x3, ord) \$

$\%$ substitution of the momenta

ham $:=\operatorname{sub}(p i x=d f(-F 3, x i), p i y=d f(-F 3, y i)$, ham $) \$$

$\%$ Hamilton-Jacobi equation

hamjac:= ham $+\operatorname{df}(\mathrm{F} 3, \mathrm{z})$ \$

$\%$ iterative solution of the equation:

for ic :=1: ord do

for $i x:=0$ : ord do for $i y:=0$ : ord do for $i 3:=0$ : ord do

if $i x+i y+i 3=i c$ then

$\ll$ term:= coeffn(coeffn(

coeffn(hamjac, pfx, ix), pfy, iy), x3, i3) \$

if term neq 0 then $\ll$

soll $:=$ solve $($ term $=0, \mathrm{df}(\mathrm{f}(\mathrm{ix}, \mathrm{iy}, \mathrm{i} 3), \mathrm{z})) \$$

sol2:= rhs (part $($ sol1, 1)) \$

sol3:= int $($ sol2, z) \$

$\mathrm{f}(\mathrm{ix}, \mathrm{iy}, \mathrm{i} 3):=\mathrm{sol} 3 \$ \gg \gg$ \$

$\%$ printing of nonvanishing terms

$\%$ results for ord $=2$ see Eqs. (15) and (21)

for ic $:=1$ : ord do

for $i x:=0$ : ord do for $i y:=0$ : ord do for $i 3:=0$ : ord do

if $i x+i y+i 3=i c$ then

if $f(i x, i y, i 3)$ neq 0 then write " $f$ ", ix, iy, i3," =",

$\operatorname{sub}(x 3=1, f(i x, i y, i 3)) \$$

end;

APPENDIX B: LIST OF ABBREVIATIONS

$c_{i(j)}, s_{i(j)}=$ Fourier coefficients

$k_{x i}=k_{x 1} \cdot i=\left(2 \pi / \lambda_{x 0}\right) \cdot i$

$\tilde{j}=1+l(j-1)$

$k_{y i, \tilde{j}}=\sqrt{k_{\tilde{j}}^{2}+k_{x i}^{2}}$

$k_{\tilde{j}}=k \cdot \tilde{j}=\left(2 \pi / \lambda_{0}\right) \cdot \tilde{j}$

$s_{x i 0}=\sin \left(k_{x i} x_{0}\right)$

$c_{x i 0}=\cos \left(k_{x i} x_{0}\right)$

$s_{x i}=\sin \left(k_{x i} x\right)$

$c_{x i}=\cos \left(k_{x i} x\right)$

$s h_{y i, \tilde{j}}=\sinh \left(k_{y i, \tilde{j}} y\right)$

$c h_{y i, \tilde{j}}=\cosh \left(k_{y i, \tilde{j}} y\right)$

$$
\begin{gathered}
s_{z \tilde{j}}=\sin \left(k_{\tilde{j}} z\right) \\
c_{z \tilde{j}}=\cos \left(k_{\tilde{j}} z\right) \\
s_{z}=\sin (k z) \\
c_{z}=\cos (k z) \\
s_{p p}=\sin \left(\varphi_{1} / 2\right) \\
c_{p p}=\cos \left(\varphi_{1} / 2\right) \\
s_{p a}=\sin \left(\varphi_{2} / 2\right) \\
c_{p a}=\cos \left(\varphi_{2} / 2\right) \\
e_{i, \tilde{j}}=\exp \left(-k_{y i, \tilde{j}} \cdot \Delta g / 2\right) \\
e_{i}=\exp \left(-k_{y i} \cdot \Delta g / 2\right) \\
B \rho=\text { beam stiffness } \\
\mathrm{L}=\text { undulator length }
\end{gathered}
$$

$x_{0}$ is the absolute value of the horizontal displacement of magnet row centers with respect to the undulator axis. $B \rho$ is the product of the magnetic field and the bending radius for a given electron energy. It characterizes the electron beam stiffness. $l$ is the period length divided by the magnet 
block length. Magnets of equal shape are assumed. For a

Halbach I undulator we have $l=4$.

\section{APPENDIX C: ANALYTIC APPLE II SCALAR POTENTIAL FOR THE UNIVERSAL MODE}

\section{Analytic scalar potential as a linear superposition of four magnet rows}

The equation represents the universal mode. The cos and sin terms are related to the transverse field expansion of a single row. The sin terms appear when the magnet blocks are not symmetric with respect to the center (e.g. cut outs for clamping). $n$ is the number of Fourier coefficients. Only one longitudinal Fourier component is regarded. The magnetic fields are derived from the scalar potential via $\vec{B}=-\vec{\nabla} \cdot V$ :

$$
\begin{aligned}
& V=\sum_{i=1}^{n}\left(V_{1 i}+V_{2 i}+V_{3 i}+V_{4 i}\right) \\
V_{1 i}= & -\left[\left(e^{+k_{y i} y} / k_{y i}\right) \cdot\left(B_{c y i} c_{x i-}-B_{s y i} s_{x i-}\right)\right. \\
& \left.+B_{0} e^{+k_{z} y} / n k_{z}\right] \cdot c_{z 1} \\
V_{2 i}= & +\left[\left(e^{-k_{y i} y} / k_{y i}\right) \cdot\left(B_{c y i} c_{x i+}+B_{s y i} s_{x i+}\right)\right. \\
& \left.+B_{0} e^{-k_{z} y} / n k_{z}\right] \cdot c_{z 2} \\
V_{3 i}= & -\left[\left(e^{+k_{y i} y} / k_{y i}\right) \cdot\left(B_{c y i} c_{x i+}+B_{s y i} s_{x i+}\right)\right. \\
& \left.+B_{0} e^{+k_{z} y} / n k_{z}\right] \cdot c_{z 3} \\
V_{4 i}= & +\left[\left(e^{-k_{y i} y} / k_{y i}\right) \cdot\left(B_{c y i} c_{x i-}-B_{s y i} s_{x i-}\right)\right. \\
& \left.+B_{0} e^{-k_{z} y} / n k_{z}\right] \cdot c_{z 4}
\end{aligned}
$$

$$
\begin{gathered}
c_{x i \pm}=\cos \left[k_{x i}\left(x \pm x_{0}\right)\right] \\
s_{x i \pm}=\sin \left[k_{x i}\left(x \pm x_{0}\right)\right] \\
c_{z 1}=\cos \left(k z+\varphi_{1} / 2\right) \\
c_{z 2}=\cos \left(k z+\varphi_{1} / 2\right) \\
c_{z 3}=\cos \left(k z-\varphi_{1} / 2+\varphi_{2}\right) \\
c_{z 4}=\cos \left(k z-\varphi_{1} / 2-\varphi_{2}\right) \\
k_{x i}=k_{x 1} \cdot i \\
k_{y i}=\sqrt{k_{x i}^{2}+k^{2}}
\end{gathered}
$$

\section{Complete analytic scalar potential of an APPLE II undulator}

Starting from C 1 we add more longitudinal Fourier components and simplify the expressions. In the following $\tilde{j}$ is the Fourier harmonic in longitudinal direction. For a pure permanent magnet configuration with four magnets per period and equal shape of all magnets only the harmonics $\tilde{j}=1,5,9 \ldots$ show up. Then the scalar potential is written as

$$
\begin{aligned}
V= & -\sum_{i=0}^{n} \sum_{j=1}^{m} 4 c_{i, \tilde{j}} \frac{1}{k_{y i, \tilde{j}}} e_{i, \tilde{j}}\left\{c _ { p p } \cdot \left[c_{x i 0} \cdot c_{x i} \cdot s h_{y i, \tilde{j}} \cdot c_{z \tilde{j}} \cdot c_{p a}^{2}-s_{x i 0} \cdot s_{x i} \cdot c h_{y i, \tilde{j}} \cdot c_{z \tilde{j}} \cdot s_{p a}^{2}\right.\right. \\
& \left.+\left(-c_{x i 0} \cdot c_{x i} \cdot c h_{y i, \tilde{j}} \cdot s_{z \tilde{j}}-s_{x i 0} \cdot s_{x i} \cdot s h_{y i, \tilde{j}} \cdot s_{z \tilde{j}}\right) \cdot c_{p a} \cdot s_{p a}\right]+s_{p p} \cdot\left[-s_{x i 0} \cdot s_{x i} \cdot c h_{y i, \tilde{j}} \cdot s_{z \tilde{j}} \cdot c_{p a}^{2}\right. \\
& \left.\left.+c_{x i 0} \cdot c_{x i} \cdot s h_{y i, \tilde{j}} \cdot s_{z \tilde{j}} \cdot s_{p a}^{2}+\left(-c_{x i 0} \cdot c_{x i} \cdot c h_{y i, \tilde{j}} \cdot c_{z \tilde{j}}-s_{x i 0} \cdot s_{x i} \cdot s h_{y i, \tilde{j}} \cdot c_{z \tilde{j}}\right) \cdot c_{p a} \cdot s_{p a}\right]\right\}
\end{aligned}
$$

\section{APPENDIX D: ANALYTIC APPLE II FIELD REPRESENTATION IN SHORT NOTATION}

From Appendix $\mathrm{C}$ the expressions of the fields are derived:

$$
\begin{aligned}
& B_{x}=\sum_{i=0}^{n} \sum_{j=1}^{m} 4 c_{i, \tilde{j}} \frac{k_{x i}}{k_{y i, \tilde{j}}} e_{i, j}[-F 5 \cdot \operatorname{cssc}-F 1 \cdot \operatorname{sccc}+F 3 \cdot(\operatorname{cscs}-\operatorname{scs} s)-F 2 \cdot \operatorname{sccs}-F 6 \cdot \operatorname{css} s+F 4 \cdot(\operatorname{cscc}-\operatorname{scsc})] \\
& B_{y}=\sum_{i=0}^{n} \sum_{j=1}^{m} 4 c_{i, j} e_{i, j}[F 5 \cdot \operatorname{cccc}-F 1 \cdot \operatorname{sssc}-F 3 \cdot(\operatorname{csss}+\operatorname{sscs})-F 2 \cdot \operatorname{sss}+F 6 \cdot \operatorname{cccs}-F 4 \cdot(\operatorname{ccsc}+\operatorname{sscc})] \\
& B_{z}=\sum_{i=0}^{n} \sum_{j=1}^{m} 4 c_{i, \tilde{j}} \frac{k}{k_{y i, \tilde{j}}} e_{i, \tilde{j}}[-F 5 \cdot \operatorname{csss}+F 1 \cdot \operatorname{sscs}-F 3 \cdot(\operatorname{cccc}+\operatorname{sssc})-F 2 \cdot \operatorname{sscc}+F 6 \cdot \operatorname{ccsc}+F 4 \cdot(\operatorname{cccs}+\operatorname{ssss})]
\end{aligned}
$$


with the abbreviations

$$
\begin{gathered}
\operatorname{cscs}=c_{x i 0} \cdot s_{x i} \cdot c h_{y i, \tilde{j}} \cdot s_{z \tilde{j}} \\
\ldots \\
F 1=c_{p p} \cdot s_{p a}^{2} \\
F 2=s_{p p} \cdot c_{p a}^{2} \\
F 3=c_{p p} \cdot c_{p a} \cdot s_{p a} \\
F 4=s_{p p} \cdot c_{p a} \cdot s_{p a}
\end{gathered}
$$

$$
\begin{aligned}
& F 5=c_{p p} \cdot c_{p a}^{2} \\
& F 6=s_{p p} \cdot s_{p a}^{2} .
\end{aligned}
$$

\section{APPENDIX E}

\section{Dynamic kicks in the universal mode in 2D}

In the following we will give only the expressions for the universal mode. The expression for the elliptical and inclined mode can be derived from the universal mode expressions by setting $F 1=F 3=F 4=F 6=0$ for the elliptical mode and $F 2=F 4=F 6=0$ for the inclined mode. We regard only the first Fourier harmonic in the longitudinal direction:

$$
\begin{aligned}
\theta_{x}= & -\frac{8 L}{(B \rho)^{2} k^{2}} \sum_{i=0}^{n} \sum_{j=0}^{n} c_{i} c_{j} e_{i} e_{j}\left(\frac { k _ { x i } } { k _ { y i } } \frac { k _ { x j } ^ { 2 } } { k _ { y j } } \left\{[-F 5 \cdot c s s-F 1 \cdot s c c+F 4 \cdot(c s c-s c s)]_{i} \cdot[-F 5 \cdot c c s+F 1 \cdot s s c\right.\right. \\
& \left.+F 4 \cdot(c c c+s s s)]_{j}+[F 3 \cdot(c s c-s c s)-F 2 \cdot s c c-F 6 \cdot c s s]_{i} \cdot[F 3 \cdot(c c c+s s s)+F 2 \cdot s s c-F 6 \cdot c c s]_{j}\right\} \\
& +k_{x j}\left\{[F 5 \cdot c c c-F 1 \cdot s s s-F 4 \cdot(c c s+s s c)]_{i} \cdot[-F 5 \cdot c s c-F 1 \cdot s c s+F 4 \cdot(c s s-s c c)]_{j}\right. \\
& \left.\left.+[-F 3 \cdot(c c s+s s c)-F 2 \cdot s s s+F 6 \cdot c c c]_{i} \cdot[F 3 \cdot(c s s-s c c)-F 2 \cdot s c s-F 6 \cdot c s c]_{j}\right\}\right) \\
\theta_{y}= & -\frac{8 L}{(B \rho)^{2} k^{2}} \sum_{i=0}^{n} \sum_{j=0}^{n} 4 c_{i} c_{j} e_{i} e_{j}\left(\frac { k _ { x i } k _ { x j } } { k _ { y i } } \left\{[-F 5 \cdot c s s-F 1 \cdot s c c+F 4 \cdot(c s c-s c s)]_{i} \cdot[-F 5 \cdot c s c-F 1 \cdot s c s\right.\right. \\
& \left.+F 4 \cdot(c s s-s c c)]_{j}+[F 3 \cdot(c s c-s c s)-F 2 \cdot s c c-F 6 \cdot c s s]_{i} \cdot[F 3 \cdot(c s s-s c c)-F 2 \cdot s c s-F 6 \cdot c s c]_{j}\right\} \\
& +k_{y j}\left\{[F 5 \cdot c c c-F 1 \cdot s s s-F 4 \cdot(c c s+s s c)]_{i} \cdot[F 5 \cdot c c s-F 1 \cdot s s c-F 4 \cdot(c c c+s s s)]_{j}\right. \\
& \left.\left.+[-F 3 \cdot(c c s+s s c)-F 2 \cdot s s s+F 6 \cdot c c c]_{i} \cdot[-F 3 \cdot(c c c+s s s)-F 2 \cdot s s c+F 6 \cdot c c s]_{j}\right\}\right)
\end{aligned}
$$

with the abbreviations

$$
c s c=c_{x i 0(x j 0)} \cdot s_{x i(x j)} \cdot c h_{y i(y j)}
$$

The sin and cos terms in the first bracket have always the indices $(i)$ and the terms in the second bracket the indices $(j)$ as indicated.

The derivatives with respect to $x$ and $y, \partial \theta_{x} / \partial x(y)$ and $\partial \theta_{y} / \partial x(y)$ can easily be evaluated.

\section{Kicks in the universal mode in the midplane}

These expressions can be used for an on-line active compensation of dynamic kicks (e.g. using the UE112 wires):

$$
\begin{aligned}
\theta_{x}= & -\frac{8 L}{(B \rho)^{2} k^{2}} \sum_{i=0}^{n} \sum_{j=0}^{n} c_{i} c_{j} e_{i} e_{j}\left(\frac{k_{x i}}{k_{y i}} \frac{k_{x j}^{2}}{k_{y j}}\left[(-F 1 \cdot s c+F 4 \cdot c s)_{i}(F 1 \cdot s s+F 4 \cdot c c)_{j}+(F 3 \cdot c s-F 2 \cdot s c)_{i}(F 3 \cdot c c+F 2 \cdot s s)_{j}\right]\right. \\
& \left.+k_{x j}\left[(F 5 \cdot c c-F 4 \cdot s s)_{i}(-F 5 \cdot c s-F 4 \cdot s c)_{j}+(-F 3 \cdot s s+F 6 \cdot c c)_{i}(-F 3 \cdot s c-F 6 \cdot c s)_{j}\right]\right) \\
\theta_{y}= & -\frac{8 L}{(B \rho)^{2} k^{2}} \sum_{i=0}^{n} \sum_{j=0}^{n} 4 c_{i} c_{j} e_{i} e_{j}\left(\frac{k_{x i} k_{x j}}{k_{y i}}\left[(-F 1 \cdot s c+F 4 \cdot c s)_{i}(-F 5 \cdot c s-F 4 \cdot s c)_{j}+(F 3 \cdot c s-F 2 \cdot s c)_{i}(-F 3 \cdot s c-F 6 \cdot c s)_{j}\right]\right. \\
& \left.+k_{y j}\left[(F 5 \cdot c c-F 4 \cdot s s)_{i}(-F 1 \cdot s s-F 4 \cdot c c)_{j}+(-F 3 \cdot s s+F 6 \cdot c c)_{i}(-F 3 \cdot c c-F 2 \cdot s s)_{j}\right]\right)
\end{aligned}
$$


using the abbreviations

$$
c s=c_{x i 0(x j 0)} \cdot s_{x i(x j)}
$$

\section{Partial derivatives of dynamic kicks on axis}

$$
\begin{aligned}
\frac{\partial \theta_{x}}{\partial x}= & -\frac{8 L}{(B \rho)^{2} k^{2}} \sum_{i=0}^{n} \sum_{j=0}^{n} c_{i} c_{j} e_{i} e_{j}\left[-F 1^{2} \frac{k_{x i}}{k_{y i}} \frac{k_{x j}^{3}}{k_{y j}} s_{i} s_{j}-F 2^{2} \frac{k_{x i}}{k_{y i}} \frac{k_{x j}^{3}}{k_{y j}} s_{i} s_{j}+F 3^{2}\left(\frac{k_{x i}^{2}}{k_{y i}} \frac{k_{x j}^{2}}{k_{y j}} c_{i} c_{j}+k_{x i} k_{x j} s_{i} s_{j}\right)\right. \\
& \left.+F 4^{2}\left(\frac{k_{x i}^{2}}{k_{y i}} \frac{k_{x j}^{2}}{k_{y j}} c_{i} c_{j}+k_{x i} k_{x j} s_{i} \cdot s_{j}\right)-F 5^{2} k_{x j}^{2} c_{i} c_{j}-F 6^{2} k_{x j}^{2} c_{i} c_{j}\right] \\
\frac{\partial \theta_{x}}{\partial y}=- & \frac{8 L}{(B \rho)^{2} k^{2}} \sum_{i=0}^{n} \sum_{j=0}^{n} c_{i} c_{j} e_{i} e_{j}\left[F 3^{2}\left(-k_{x i} \frac{k_{x j}^{2}}{k_{y j}} s_{i} c_{j}+k_{y i} k_{x j} F 3^{2} c_{i} s_{j}\right)+F 4^{2}\left(-k_{x i} \frac{k_{x j}^{2}}{k_{y j}} s_{i} c_{j}+k_{y i} k_{x j} c_{i} s_{j}\right)\right. \\
+ & \left.F 1 \cdot F 5\left(\frac{k_{x i}}{k_{y i}} k_{x j}^{2} s_{i} c_{j}-k_{x j} k_{y j} c_{i} s_{j}\right)+F 2 \cdot F 6\left(\frac{k_{x i}}{k_{y i}} k_{x j}^{2} s_{i} c_{j}-k_{x j} k_{y j} c_{i} s_{j}\right)\right] \\
& \left.+F 1 \cdot F 5\left(\frac{k_{x i}}{k_{y i}} k_{x j}^{2} s_{i} c_{j}-k_{x j} k_{y j} c_{i} s_{j}\right)+F 2 \cdot F 6\left(\frac{k_{x i}}{k_{y i}} k_{x j}^{2} s_{i} c_{j}-k_{x j} k_{y j} c_{i} s_{j}\right)\right] \\
\frac{\partial \theta_{y}}{\partial y}= & -\frac{8 L}{(B \rho)^{2} k^{2}} \sum_{i=0}^{n} \sum_{j=0}^{n} 4 c_{i} c_{j} e_{i} e_{j}\left[F 3 ^ { 2 } \left(-\frac{k_{x i}^{2}}{k_{y i}} k_{x j} \sum_{j=0}^{n} 4 c_{i} c_{j} s_{j}+k_{x i} e_{j}\left[\frac{k_{x i}}{k_{y i}} k_{x j} k_{y j} c_{j}\right)+F 1^{2} s_{i} s_{j}+F 2^{2} \frac{k_{x i}}{k_{y i}} k_{x j} k_{y j} s_{i} s_{j}+F 3^{2}\left(k_{x i} k_{x j} s_{i} s_{j}+k_{y i} k_{y j} c_{i} c_{j}\right)\right.\right. \\
& \left.\left.+F 4^{2}\left(k_{x i} k_{x j} s_{i} s_{j}+k_{y i} k_{y j} c_{i} c_{j}\right)+F 5^{2} k_{y j}^{2} c_{i} c_{j}+F 6^{2} k_{y j}^{2} c_{i} c_{j}\right)\right] .
\end{aligned}
$$

During phase motion of an APPLE II undulator (e.g. switching of helicity at the same energy) only the functions F1-F6 change their value. The other parameters are constant. The functions F1-F6 do not depend on $i$ and $j$ and they can be factored out. Thus, the double sums can be evaluated beforehand and during the phasing only F1-F6 have to be evaluated for the determination of the dynamic kicks and coupling terms.

[1] J. Safranek, C. Limborg, A. Terebilo, K. I. Blomqvist, P. Elleaume, and Y. Nosochkov, in Proceedings of the European Particle Accelerator Conference, Vienna, 2000 (EPS, Geneva, 2000), pp. 295-297.

[2] L. Elias et al., Phys. Rev. Lett. 36, 717 (1976).

[3] D. Deacon et al., Phys. Rev. Lett. 38, 892 (1977).

[4] P. Elleaume, Nucl. Instrum. Methods Phys. Res., Sect. A 291, 371 (1990).

[5] B. Diviacco et al., Nucl. Instrum. Methods Phys. Res., Sect. A 292, 517 (1990).

[6] T. Hara et al., J. Synchrotron Radiat. 5, 426 (1998).

[7] S. Sasaki, Nucl. Instrum. Methods Phys. Res., Sect. A 347, 83 (1994).

[8] J. Bahrdt et al., Proceedings of the 26th International FEL Conference, Trieste, Italy, 2004, pp. 610-613, available online at http://www.JACoW.org.

[9] A. Temnykh, Phys. Rev. ST Accel. Beams 11, 120702 (2008).
[10] A. Temnykh, in Proceedings of the 20th Particle Accelerator Conference, Portland, OR, 2003 (IEEE, New York, 2003), pp. 1026-1028.

[11] P. Elleaume, in Proceedings of the 1992 European Particle Accelerator Conference, Berlin, Germany (European Physical Society, 1992), pp. 661-663.

[12] H. Lustfeld et al., in Proceedings of the Linear Accelerator Conference, Seeheim, Germany, 1984 (Report No. GSI84-11).

[13] J. Bahrdt and G. Wüstefeld, "A New Tracking Routine for Particles in Undulator and Wiggler Fields," Parts I, II, and III, BESSY TB No. 168 and TB No. 163, BESSY, Berlin, Germany, 1990.

[14] J. Bahrdt and G. Wüstefeld, in Proceedings of the 1991 Particle Accelerator Conference, San Francisco, CA, 1991 (IEEE, New York, 1991), pp. 266-268.

[15] J. Bahrdt and G. Wüstefeld, in Proceedings of the 1992 European Particle Accelerator Conference, Berlin, Germany (European Physical Society, 1992), pp. 670-672. 
[16] G. Wüstefeld, in Proceedings of the Particle Accelerator Conference, Dallas, TX, 1995 (IEEE, New York, 1995), pp. 2868-2870.

[17] J. Bahrdt, M. Scheer, and G. Wüstefeld, in Proceedings of the 10th European Particle Accelerator Conference, Edinburgh, Scotland, 2006 (EPS-AG, Edinburgh, Scotland, 2006), pp. 3562-3564.

[18] J. Bahrdt, W. Frentrup, A. Gaupp, M. Scheer, and G. Wüstefeld, in Proceedings of the 2007 Particle Accelerator Conference, Albuquerque, New Mexico (IEEE, New York, 2007), pp. 941-943.

[19] M. Scheer and G. Wüstefeld, in Proceedings of the 1997 Particle Accelerator Conference, Vancouver, BC (IEEE, New York, 1998), pp. 1418-1420.

[20] M. Scheer, Ph.D. thesis, Humboldt University Berlin, 2008.

[21] R.D. Ruth, in Proceedings of the 1983 Particle Accelerator Conference, Santa Fe, NM (IEEE, New York, 1983), pp. 2669-2671.

[22] R.L. Warnock, in Proceedings of the 1989 Particle Accelerator Conference, Chicago, IL (IEEE, New York, 1989), pp. 1322-1324.

[23] D. A. Edwards and M. J. Syphers, The Hamiltonian Formalism, Wiley Series in Beam Physics and Accelerator Technology (Wiley, New York, 1993).

[24] H. Goldstein, Klassische Mechanik (Akademische Verlagsgesellschaft, Frankfurt, Germany, 1963).

[25] A.C. Hearn, REDUCE 3.5, A General Purpose Algebra System (RAND, Santa Monica, California, 1993), http:// www.uni-koeln.de/REDUCE/.

[26] J. Bahrdt, R. Follath, W. Frentrup, A. Gaupp, and M. Scheer, in Proceedings of the 2009 Synchrotron Radiation Instrumentation Conference, Melbourne, Victoria, Australia, AIP Conf. Proc. No. 1234 (AIP, New York, 2009), pp. 235-238.

[27] F. Schäfers, H.-C. Mertins, A. Gaupp, W. Gudat, M. Mertin, I. Packe, F. Schmolla, S. Di Fonzo, G. Soullié, W. Jark, R. Walker, X. Le Cann, R. Nyholm, and M. Eriksson, Appl. Opt. 38, 194074 (1999).

[28] J. Bahrdt et al., in Proceedings of the 2009 Particle Accelerator Conference, Vancouver, BC, Canada (IEEE, Piscataway, NJ, 2009), pp. 1162-1164.

[29] J. Bahrdt, H.-J. Bäcker, W. Frentrup, A. Gaupp, M. Scheer, B. Schulz, U. Englisch, and M. Tischer, in Proceedings of the 11th European Particle Accelerator
Conference, Genoa, 2008 (EPS-AG, Genoa, Italy, 2008), pp. 2219-2221.

[30] J. Bahrdt, H.-J. Bäcker, W. Frentrup, A. Gaupp, S. Gottschlich, C. Kuhn, M. Scheer, B. Schulz, M. Gast, U. Englisch, A. Schöps, and M. Tischer, in Proceedings of the 2009 Synchrotron Radiation Instrumentation Conference, Melbourne, Victoria, Australia (Ref. [26]), pp. 503-506.

[31] R. Carr, Nucl. Instrum. Methods Phys. Res., Sect. A 306, 391 (1991).

[32] T. Schmidt and D. Ziemoch, in Proceedings of the 2006 Synchrotron Radiation Instrumentation Conference, Daegu, South Korea, AIP Conf. Proc. No. 879 (AIP, New York, 2006), pp. 400-403.

[33] T. Schmidt and D. Ziemoch, in Proceedings of the 2006 Synchrotron Radiation Instrumentation Conference, Daegu, South Korea (Ref. [32]), pp. 404-407.

[34] K. Halbach, Nucl. Instrum. Methods 187, 109 (1981).

[35] P. Elleaume et al., in Proceedings of the 1997 Particle Accelerator Conference, Vancouver, BC (Ref. [19]), pp. 3509-3511.

[36] O. Chubar et al., J. Synchrotron Radiat. 5, 481 (1998).

[37] J. Bahrdt et al., in Proceedings of the 2006 Synchrotron Radiation Instrumentation Conference, Daegu, South Korea (Ref. [32]), pp. 315-318.

[38] R. Walker, Nucl. Instrum. Methods Phys. Res., Sect. A 335, 328 (1993).

[39] D. C. Quimby et al., Nucl. Instrum. Methods Phys. Res., Sect. A 285, 281 (1989).

[40] S. C. Gottschalk et al., Nucl. Instrum. Methods Phys. Res., Sect. A 296, 579 (1990).

[41] B. Diviacco et al., Nucl. Instrum. Methods Phys. Res., Sect. A 368, 522 (1996).

[42] P. Elleaume et al., Synchrotron Radiat. News 8, 18 (1995).

[43] J. Bahrdt et al., Nucl. Instrum. Methods Phys. Res., Sect. A 516, 575 (2004).

[44] S. Marks et al., Proceedings of the 1999 PAC, New York, NY, USA, pp. 162-164.

[45] J. Bahrdt et al., in Proceedings of the 11th European Particle Accelerator Conference, Genoa, 2008 (Ref. [29]), pp. 2222-2224.

[46] J. Chavanne et al., in Proceedings of the European Particle Accelerator Conference, Vienna, 2000 (EPS, Geneva, 2000), pp. 2346-2348. 